\title{
The Hypergroup Property and Representation of Markov Kernels
}

\author{
Dominique Bakry ${ }^{1}$ and Nolwen Huet ${ }^{2}$ \\ 1 IUF and Institut de Mathématiques, Université de Toulouse and CNRS \\ 118, route de Narbonne, 31400 Toulouse, France \\ dominique.bakry@math.univ-toulouse.fr \\ 2 Institut de Mathématiques, Université de Toulouse and CNRS \\ 118, route de Narbonne, 31400 Toulouse, France \\ nolwen.huet@math.univ-toulouse.fr
}

Summary. For a given orthonormal basis $\left(f_{n}\right)$ on a probability measure space, we want to describe all Markov operators which have the $f_{n}$ as eigenvectors. We introduce for that what we call the hypergroup property. We study this property in three different cases.

On finite sets, this property appears as the dual of the GKS property linked with correlation inequalities in statistical mechanics. The representation theory of groups provides generic examples where these two properties are satisfied, although this group structure is not necessary in general.

The hypergroup property also holds for Sturm-Liouville bases associated with logconcave symmetric measures on a compact interval, as stated in Achour-Trimèche's theorem. We give some criteria to relax this symmetry condition in view of extensions to a more general context.

In the case of Jacobi polynomials with non-symmetric parameters, the hypergroup property is nothing else than Gasper's theorem. The proof we present is based on a natural interpretation of these polynomials as harmonic functions and is related to analysis on spheres. The proof relies on the representation of the polynomials as the moments of a complex variable.

\section{Contents}

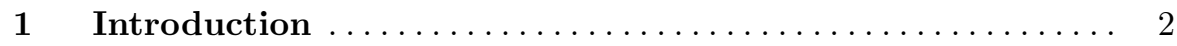

2 The finite case $\ldots \ldots \ldots \ldots \ldots \ldots \ldots \ldots \ldots \ldots \ldots \ldots \ldots \ldots \ldots$

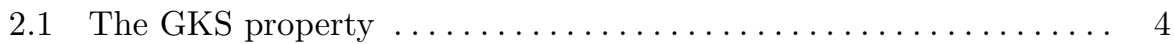

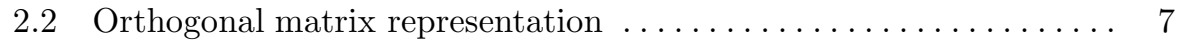

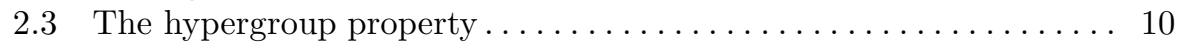

2.4 Markov operators as convolutions $\ldots \ldots \ldots \ldots \ldots \ldots \ldots \ldots \ldots \ldots$

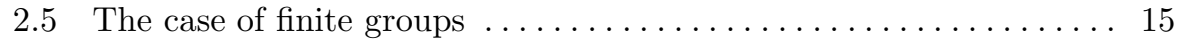


2.6 On the GKS inequalities $\ldots \ldots \ldots \ldots \ldots \ldots \ldots \ldots \ldots \ldots \ldots$

3 The hypergroup property in the infinite setting ........ 20

3.1 Markov sequences associated with a UOB . . . . . . . . . . . 20

3.2 The hypergroup property .......................... 22

4 Sturm-Liouville bases and Achour-Trimèche's theorem ... 24

4.1 The natural UOB associated with a measure on a compact interval 24

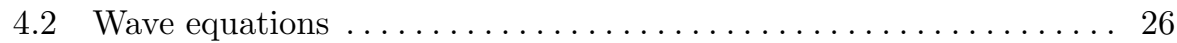

4.3 Achour-Trimèche's theorem and wave equations............ 28

4.4 Other representations of the solutions of the wave equation ...... 31

4.5 More about the solutions of the wave equation (4) .......... 36

5 The case of Jacobi polynomials: Gasper's theorem ....... 40

5.1 Jacobi polynomials .............................. 40

5.2 Gasper's result ............................... 42

5.3 The special case of ultraspherical polynomials $(p=q \geq 1) \ldots \ldots 43$

5.4 The case of dissymmetric Jacobi polynomials $(q>p>1) \ldots \ldots .45$

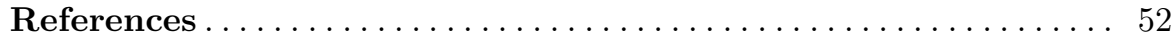

\section{Introduction}

In a number of situations, Markov operators appear to be a wonderful tool to provide useful information on a given measured space. Let us for example mention heat kernel methods to prove functional inequalities like Sobolev or Log-Sobolev inequalities, or Cauchy kernels to prove boundness results on Riesz transforms in $L^{p}$. Heat kernels are widely used in Riemannian geometry and statistical mechanics, while Poisson, Cauchy and other kernels had been proved useful in other contexts related to classical harmonic analysis (see $[9,5,6,7,11,10,20,28,29,2,38]$ for example to see the action of different families of semigroups in various contexts).

It seems therefore interesting to describe all Markov kernels associated with a given structure. In what follows, we shall consider a probability space $(E, \mathcal{E}, \mu)$ on which is given an orthonormal $L^{2}(\mu)$ basis $\mathcal{F}=\left(f_{0}, \ldots, f_{n}, \ldots\right)$, where we impose $f_{0}=1$. Such a basis shall be called a unitary orthonormal basis (UOB in short). In general our basis $\mathcal{F}$ shall be real, but we do not exclude to consider complex bases.

We may then try to describe the Markov operators defined from a family of probability measures $k(x, d y)$ by

$$
f \mapsto K(f)=\int_{E} f(y) k(x, d y)
$$

which are symmetric in $L^{2}(\mu)$ and have the functions $f_{n}$ as eigenvectors. In other words, we want to define the linear operator $K$ from 


$$
K\left(f_{n}\right)=\lambda_{n} f_{n}
$$

and try to describe for which sequences $\left(\lambda_{n}\right)$ this operator is a Markov kernel. We shall call these sequences Markov sequences (MS's in short) associated with the UOB $\mathcal{F}$.

For a general basis $\mathcal{F}$, this is quite impossible. But many bases which appear in natural examples have a special property, which we call the hypergroup property, under which one is able to describe all Markov sequences associated with the UOB $\mathcal{F}$.

This expository paper is not intended to be a complete account of the general theory of hypergroups, for which we may for example refer to the complete treatise [13]. In fact, we just extracted from this theory what is useful for our purpose. More precisely, we concentrated on the fundamental aspect which we are interested in, that is the possibility of describing all Markov kernels associated with our basis $\mathcal{F}$.

The paper is organized as follows.

In the first part, we present the case of finite sets, where the hypergroup property appears as the dual property of a more natural condition on the basis $\mathcal{F}$, namely the positivity of the multiplication coefficients. This property is called the GKS property in [8] because of its links with some famous correlation inequalities in statistical mechanics, and we keep this notation. These correlation inequalities did in fact motivate our efforts in this direction (see Paragraph 2.6). Many examples come from the representation theory of groups, but we propose a systematic exposition. The hypergroup property provides a convolution operation on the set of probability measures on the space, and the Markov kernels may be represented as the convolution with some given measure. In this situation, the hypergroup property appears as a special property of an orthogonal matrix. We shall see that in fact there are many situations where no group structure holds and where nevertheless the hypergroup property holds.

The second part is devoted to the presentation of Achour-Trimèche's theorem, which states this property for the basis of eigenvectors of a SturmLiouville operator with Numen boundary conditions, associated with a logconcave symmetric measure on a compact interval. The original AchourTrimèche's theorem was not stated exactly in the same way (see [1]), but his argument carries over very easily to our context. We give a complete proof of this result, since to our knowledge this proof was never published. We tried to relax the symmetry condition on the measure, and provided various rather technical extensions of the theorem. But we did not succeed to extend Achour-Trimèche's theorem to a wider class of measures which would include the case of Gasper's theorem on Jacobi polynomials studied in the next chapter. The real motivation of this effort is that in general, log-concave measures on $\mathbb{R}$ or on an interval serve as a baby model in Riemannian geometry for manifolds with non-negative Ricci curvature. Unfortunately, the symmetry condition does not seem to have any natural interpretation. 
In the last part, we present Gasper's theorem, which states hypergroup property for the Jacobi polynomials. We present a proof which relies on geometric considerations on the spheres when the parameters are integers, and which easily extends to the general case. We follow Koornwinder's proof of the result, and give a natural interpretation of Koornwinder's formula (Lemma 5.2 ) which represents those polynomials as the moments of some complex random variable. We found after the redaction of this part that the interpretation of Jacobi polynomials as harmonic functions was already known from specialists (see $[14,23,35]$ ) but it seems that it was not directly used to prove this integral representation formula. We hope that this simple interpretation may provide other examples for similar representation in other contexts.

\section{The finite case}

In this section, we restrict ourselves to the case of finite sets, since in this context most of the ideas underlying the general setting are present, and we so avoid the analytic complexity of the more general cases that we shall study later on.

\subsection{The GKS property}

In what follows, we assume that our space is a finite set

$$
E=\left\{x_{0}, \ldots, x_{n}\right\},
$$

endowed with a probability measure

$$
\mu=\left(\mu\left(x_{0}\right), \ldots, \mu\left(x_{n}\right)\right) .
$$

We denote by $L^{2}(\mu)$ the space of real functions on $E$, and we assume the existence of a real basis

$$
\mathcal{F}=\left(f_{0}, \ldots, f_{n}\right)
$$

with $f_{0}=1$. We suppose here that for any $x \in E, \mu(x)>0$.

We shall write $\langle f\rangle$ for $\int f d \mu$ and $\langle f, g\rangle$ for $\int f g d \mu$.

The algebra structure of the set of functions is reflected in the multiplication tensor $\left(a_{i j k}\right)$ for which

$$
f_{i} f_{j}=\sum_{k} a_{i j k} f_{k} .
$$

We therefore have

$$
a_{i j k}=\left\langle f_{i} f_{j} f_{k}\right\rangle,
$$

and we see that the tensor $\left(a_{i j k}\right)$ is symmetric in $(i j k)$. It has also another property which reflects the fact that the multiplication is associative. 
Definition 2.1. We shall say that $\mathcal{F}$ has the property GKS if all coefficients $\left(a_{i j k}\right)$ are non-negative.

This notation comes from the GKS inequality in statistical mechanics that we shall describe at the end of this section.

Observe that $a_{i j 0}=\delta_{i j}$.

Many natural bases $\mathcal{F}$ share this property. For example, consider the hypercube $E=\{-1,1\}^{N}$, with the uniform measure on it. Let $\omega_{i}$ denote the $i$-th coordinates

$$
\omega=\left(\omega_{1}, \ldots, \omega_{n}\right) \mapsto \omega_{i},
$$

and, for $A \subset\{1, \ldots, N\}$

$$
\omega_{A}=\prod_{i \in A} \omega_{i}, \omega_{\emptyset}=1
$$

Then,

$$
\mathcal{F}=\left\{\omega \mapsto \omega_{A}, A \subset\{1, \ldots, N\}\right\}
$$

is a $\mathrm{UOB}$ of $(E, \mu)$. Since

$$
\omega_{A} \omega_{B}=\omega_{A \Delta B}
$$

it has the GKS property.

(We shall see later that this is a special case of a generic situation in finite groups).

Although we are here mainly interested in the case of a real basis, there are many natural complex GKS bases, issued in general from the representation theory of finite groups (see Paragraph 2.5 later). If the basis is complex, we shall still require that the multiplication coefficients are non-negative real numbers, which means that

$$
a_{i j k}=\left\langle f_{i} f_{j} \bar{f}_{k}\right\rangle \geq 0
$$

for any $(i, j, k)$.

In what follows, we only consider real GKS bases, although the next result remains probably true in the complex setting.

Proposition 2.1. If a UOB has the GKS property, then there exists a unique point $x_{0}$ on which every $f_{i}\left(x_{0}\right)$ is maximal. Moreover, for any $i$ and any $x$, $\left|f_{i}\right|(x) \leq f_{i}\left(x_{0}\right)$, and at this point $x_{0}, \mu\left(x_{0}\right)$ is minimal.

Proof. Let us say that a function $f: E \mapsto \mathbb{R}$ is GKS if for any $i=0, \ldots, n$, $\left\langle f f_{i}\right\rangle \geq 0$. In other words, $f$ is written with non-negative coefficients in the basis $\mathcal{F}$.

We shall say that a set $K \subset E$ is GKS if $\mathbb{1}_{K}$ is a GKS function. We shall say that a point $x$ is GKS if $\{x\}$ is a GKS set.

We shall see that there is only one GKS point.

Remark first that the sum of two GKS functions is GKS and that, thanks to the GKS property of $\mathcal{F}$, the product of two GKS functions is GKS. Moreover 
a limit of GKS functions is GKS. Observe also that a GKS function has always a non-negative integral with respect to $\mu$ since $f_{0}=1$.

Let us consider a non-zero GKS function $f$ and consider $m=\max _{x \in E}|f|$. We see first that $\{f=m\} \neq \emptyset$.

For this, assume the contrary, that is that $f=-m$ on $|f|=m$. Since $m>$ 0 , we see that $f^{2 p+1} / m^{2 p+1}$ is a GKS function, and converges to $-\mathbb{1}_{\{f=-m\}}$. Since a GKS function has a non-negative integral, this is impossible. Using the same argument, we see that

$$
\frac{1}{2}\left(\lim _{n} \frac{f^{2 n}}{m^{2 n}}+\lim _{n} \frac{f^{2 n+1}}{m^{2 n+1}}\right)=\mathbb{1}_{\{f=m\}}
$$

is a GKS function.

Therefore, the set $\{f=m\}$ is a GKS set, and there are non-trivial GKS sets.

Moreover, for any GKS function, the set $\{f=\max |f|\}$ is GKS.

Let $E_{1}$ be a nonempty GKS set, minimal for the inclusion. Then, for any GKS function $f, g=\mathbb{1}_{E_{1}} f$ is GKS. If $g$ is not 0 , then its maximum is attained on a subset $E_{2}$ of $E_{1}$ which is again GKS. Since $E_{1}$ is minimal, we have $E_{2}=E_{1}$.

Therefore, for any GKS function, its restriction $g$ to $E_{1}$ is either 0 on $E_{1}$ or constant (and equal to the maximum of $g$ ). In any case, $f$ is constant on $E_{1}$. Since this applies to every function $f_{i}$ in $\mathcal{F}$, and since $\mathcal{F}$ is a basis, every function is constant on $E_{1}$ and therefore $E_{1}$ is reduced to a single point $\left\{x_{0}\right\}$.

The same proof shows that any GKS set contains a GKS point.

For a GKS point $x_{0}, f_{i}\left(x_{0}\right)=\left\langle f_{i} \mathbb{1}_{x_{0}}\right\rangle / \mu\left(x_{0}\right) \geq 0$.

Then, consider two distinct points $x_{0}$ and $x_{1}$, and write

$$
\frac{\mathbb{1}_{x_{0}}}{\mu\left(x_{0}\right)}=\sum_{k} f_{k}\left(x_{0}\right) f_{k}, \frac{\mathbb{1}_{x_{1}}}{\mu\left(x_{1}\right)}=\sum_{k} f_{k}\left(x_{1}\right) f_{k} .
$$

Writing the product, we see that

$$
0=\frac{\mathbb{1}_{x_{0}} \mathbb{1}_{x_{1}}}{\mu\left(x_{0}\right) \mu\left(x_{1}\right)}=\sum_{i j k} a_{i j k} f_{i}\left(x_{0}\right) f_{j}\left(x_{1}\right) f_{k},
$$

with the multiplication coefficients $a_{i j k}$.

So we see that for any pair of distinct points, and for any $k$,

$$
\sum_{i j} f_{i}\left(x_{0}\right) f_{j}\left(x_{1}\right) a_{i j k}=0 .
$$

Suppose then that $x_{0}$ and $x_{1}$ are GKS points. In the previous sum, all coefficients are non-negative. Therefore, for any $(i, j, k)$

$$
a_{i j k} f_{i}\left(x_{0}\right) f_{j}\left(x_{1}\right)=0 .
$$


If we apply that with $i=0$ and $j=k$, we see that $f_{j}\left(x_{1}\right)=0$, for any $i$. This is impossible since then any function would take the value 0 in $x_{1}$. So there is a unique GKS point.

Let $x_{0}$ be this unique GKS point. Any GKS set contains $x_{0}$. Since for any GKS function, the set where $f$ is maximum is GKS, any GKS function attains its maximum at $x_{0}$.

It remains to show that $\mu$ is minimal at $x_{0}$. For this, observe that for any point $x$, the function

$$
f_{x}=\mu(x) \mathbb{1}_{x_{0}}-\mu\left(x_{0}\right) \mathbb{1}_{x}
$$

is GKS (this comes from the fact that each $f_{i}$ is maximal at $x_{0}$ ). Therefore, the maximum value of $\left|f_{x}\right|$ is attained in $x_{0}$, which gives the result.

\subsection{Orthogonal matrix representation}

Consider the matrix

$$
\left(O_{i j}\right)=\left(\sqrt{\mu\left(x_{i}\right)} f_{j}\left(x_{i}\right)\right),
$$

we see easily that the matrix $\left(O_{i j}\right)$ is a $(n+1) \times(n+1)$ orthogonal matrix with positive first column. Conversely, any such matrix may be associated with a UOB on a finite set with measure $\mu$ given by

$$
\mu\left(x_{i}\right)=O_{i 0}^{2} .
$$

Therefore, there is a one to one correspondence between the set of orthogonal matrices with positive first column, and the set of finite probability spaces, whose probability has everywhere positive weight, endowed with a UOB. (In fact, this is not completely true, since we would not distinguish between bases given in different orders, provided that the first element is 1 , which identifies the set of UOBs with a quotient of a the set of orthogonal matrices through a permutation of rows and columns.)

The GKS property may be translated into the following property on such an orthogonal matrix:

$$
\forall j, k, l, \sum_{i} \frac{O_{i j} O_{i k} O_{i l}}{O_{i 0}} \geq 0 .
$$

The transposed of an orthogonal matrix is orthogonal, and we just saw that an orthogonal matrix which has the GKS property also has a non-negative row (corresponding to the row where the first column is minimal according to Proposition 2.1). We may of course rearrange the labelling of the points in such a way that this row is the first one. Then, the situation is completely symmetric.

We shall then consider the squares of terms in the first row as a probability measure on the dual set $\{0,1, \ldots, n\}$ :

$$
\nu(i)=\mu\left(x_{0}\right) f_{i}^{2}\left(x_{0}\right) .
$$


Thanks to the fact that the functions $f_{i}$ are maximal at $x_{0}$ and that this maximum must be larger than 1 (since $\int f_{i}^{2} d \mu=1$ ), the dual measure is also minimum at 0 .

As an application, we have the following.

Proposition 2.2. If a real GKS basis exists for the uniform measure on some finite set $E$, then the cardinal of $E$ must be $2^{k}$ for some $k$, and this basis is the canonical basis $\left(\omega_{A}\right)$ of the characters of the group $(\mathbb{Z} / 2 \mathbb{Z})^{k}$.

To see this, we first observe that the dual measure is uniform too. In fact, for the matrix $\left(O_{i j}\right)$, we have $O_{00}=1 / \sqrt{n+1}$, where $n+1$ is the number of points in the space, and since the first row is positive and has minimum value $1 / \sqrt{n+1}$, it must be constant since the sum of the squares of its coefficients is 1 .

Now, if we multiply the matrix $O$ by $\sqrt{n+1}$, then we see that in each column, the maximum value of the coefficients is attained on the first row and is equal to 1 . Since the sum of all the squares of the coefficients in a given column must add to $n+1$, this shows that in any column, the coefficients must take only the values \pm 1 .

Those matrices (with entries \pm 1 and orthogonal lines) are called Hadamard matrices (cf $[30,37,33])$. If $n+1=2^{k}$ for some $k$, such matrices are given by the basis $\left(\omega_{A}\right)$ on $\{-1,1\}^{k}$ and satisfy the GKS property. It is known that the order of a Hadamard matrix must be 1,2 , or $4 k$, and it is an open problem to find such matrices for all $k$ (the lowest $k$ for which no Hadamard matrix of order $4 k$ is known is $k=167$ since [33]). Nevertheless, the following proposition will prove the result of Proposition 2.2.

Proposition 2.3. If a Hadamard matrix has the GKS property, then it must be of order $2^{k}$ for some $k$, and, up to permutation, it is the matrix of the canonical basis $\left(\omega_{A}\right)$ of the group $\{-1,1\}^{k}$.

Proof. The case of a set of size 2 is trivial, and we therefore assume that the size of the matrix is at least 3 . To fix the idea, consider a matrix $M$ of order $n+1$ with entries \pm 1 , with orthogonal columns. Call $f_{i}$ the column vectors and $\hat{f}_{i}=f_{i} / \sqrt{n+1}$ the normalized ones, so that $M=\left(f_{i}\left(x_{j}\right)\right)_{0 \leq i, j \leq n}$. As usual, let us denote by $\langle f\rangle$ the mean value of a function $f$ with respect to the normalized uniform measure. We suppose, which is possible up to reordering, that all the entries of the first line and the first column are +1 . The GKS property says that

$$
\left\langle f_{i} f_{j} f_{k}\right\rangle \geq 0
$$

for any $(i, j, k)$.

First, since $\left\langle f_{i}\right\rangle=0$ for any $i \geq 1$, there must be as many 1 and -1 in each column, and therefore $n+1$ is even. Let $A$ be the set of points where $f_{1}=1$. Let $i \geq 2$, and let $q$ be the number of points in $x \in A$ such that $f_{i}(x)=1$, and $r$ be the number of points in $A^{c}$ where $f_{i}(x)=1$. Writing $\left\langle f_{i}\right\rangle=0$ and $\left\langle f_{1} f_{i}\right\rangle=0$, we get 


$$
q+r=p, q=r,
$$

which shows that $p$ is even and also that, when $i \geq 2$,

$$
\left\langle\mathbb{1}_{A} f_{i}\right\rangle=0
$$

This is the generic argument which shows that Hadamard matrices have order $4 k$. We shall now make use of the GKS property. Write

$$
\mathbb{1}_{A} f_{i}=\sum_{l} a_{i l} f_{l}
$$

We have

$$
a_{i l}=\left\langle\mathbb{1}_{A} \hat{f}_{i} \hat{f}_{l}\right\rangle=\frac{1}{n+1}\left\langle\mathbb{1}_{A} f_{i} f_{l}\right\rangle
$$

and therefore the matrix $A=\left(a_{i l}\right)$ is symmetric. As it is the matrix of a projector, its eigenvalue are 0 or 1 . The dimension of the eigenspace associated to 1 is $p$, since the eigenspace is generated by $\left(\mathbb{1}_{\{x\}}, x \in A\right)$. Also, $A$ is a GKS set because it is the set where $f_{1}$ attains it's maximum (cf proof of Proposition 2.1, Page 6). This implies that all the entries of $A$ are not negative. Moreover, if we look at the values of the functions at $x_{0}$, it holds $\sum_{j} a_{i j}=1$.

Therefore, the matrix $A$ is Markovian. There are no transitory points since $A$ is symmetric. The number of recurrence classes for such a matrix is the multiplicity of 1 as eigenvector, here $p$, so there are exactly $p$ recurrence classes, and no recurrence class is reduced to a single point, since $a_{i i}=1 / 2<1$. So every recurrence class has exactly two points. For example, $\{0,1\}$ form a recurrence class. On each line of the matrix, there are exactly two places where $a_{i j} \neq 0$. The values of those entries are then $1 / 2$, since $a_{i i}=1 / 2$. If we choose two distinct indices $i$ and $j$ in two different recurrent classes, then $a_{i j}=0$. This means that $\left\langle\mathbb{1}_{A} f_{i} f_{j}\right\rangle=0$.

Choose now one index in every recurrence class (say the even indices to fix the ideas, which is possible up to reordering of the columns). Then, the functions $g_{i}=\mathbb{1}_{A} f_{2 i}$ form an orthogonal Hadamard GKS matrix of order $p=(n+1) / 2$, and we may now use induction to see that the order must be $2^{k}$ for some $k$.

To see that the unique basis such basis in dimension $2^{k}$ is given by the canonical basis, it is enough to observe that if $i$ and $\sigma(i)$ are in the same recurrence class for the matrix $A$, then we have

$$
\mathbb{1}_{A} f_{i}=\frac{1}{2}\left(f_{i}+f_{\sigma(i)}\right)=\mathbb{1}_{A} f_{\sigma(i)},
$$

and also

$$
\mathbb{1}_{A^{c}} f_{i}=-\mathbb{1}_{A^{c}} f_{\sigma(i)} .
$$

Then an easy induction leads to the result. 
Nevertheless, unlike real bases, we shall see in Paragraph 2.5 that there always exists a complex GKS basis on any finite set with uniform measure.

On two points, an easy computation shows that a two dimensional orthogonal matrix having the GKS property must be

$$
\left(\begin{array}{cc}
\cos \theta & \sin \theta \\
\sin \theta & -\cos \theta
\end{array}\right)
$$

with $\theta \in[\pi / 4, \pi / 2)$. In fact, in dimension 2 , given the measure (giving two distinct positive masses on the two points), there are exactly two UOB, and only one such that the unique non-constant function is maximal on the point with minimal mass (a necessary condition to have the GKS property as we saw). In this situation, any GKS matrix is symmetric, and the set of orthogonal matrices having the GKS property is connected.

On three points, the situation is more complicated. We saw for example that there are no real GKS basis when the measure is uniform. The set of real GKS UOBs on three points is connected, and one may see that the maximum value of $\mu\left(x_{0}\right)$ for which there exists a real GKS basis is $\mu\left(x_{0}\right)=1 / 4$, and in this case the probability measure is $(1 / 4,1 / 4,1 / 2)$ and the unique GKS basis is obtained taking the real parts of the characters in the group $\mathbb{Z} / 4 \mathbb{Z}$ (we shall see later in Paragraph 2.5 how to associate complex or real GKS bases with any finite group). There is also a complex GKS basis with the uniform measure (the characters of the group $\mathbb{Z} / 3 \mathbb{Z}$ ).

\subsection{The hypergroup property}

As we saw before, when we have the GKS property, the situation is completely symmetric and we may consider the dual property. This is the hypergroup property.

Definition 2.2. We shall say that the $U O B \mathcal{F}$ of $L^{2}(\mu)$ satisfies the hypergroup property (HGP in short) at point $x_{0}$ if for any $(x, y, z) \in E^{3}$,

$$
K(x, y, z)=\sum_{i} \frac{f_{i}(x) f_{i}(y) f_{i}(z)}{f_{i}\left(x_{0}\right)} \geq 0 .
$$

Of course, this supposes that for any $i, f_{i}\left(x_{0}\right) \neq 0$.

We do not require the GKS property to hold in the definition of the HGP property. We shall see later (at the end of Paragraph 2.5, Page 18) that we can find bases with HGP property and not GKS property, and the reverse. On the other hand, we shall see that in groups, the natural basis always share both property.

Remark. Since we may always change for any $i \geq 1 f_{i} \in \mathcal{F}$ into $-f_{i}$, and still get a UOB, and that this operation does not change the hypergroup property, we see that we may always assume that $f_{i}\left(x_{0}\right)>0$. 
To see the duality with the previous situation, let us enumerate the points in $E$ starting from $x_{0}$, and recall our orthogonal matrix $O$ with $O_{i j}=\sqrt{\mu\left(x_{i}\right)} f_{j}\left(x_{i}\right)$.

Then, under the GKS condition, $O$ has non-negative first line and first column. Recall that the GKS property may be written as

$$
\forall j, k, l, \sum_{i} \frac{O_{i j} O_{i k} O_{i l}}{O_{i 0}} \geq 0 .
$$

while the HGP property writes

$$
\forall j, k, l, \sum_{i} \frac{O_{j i} O_{k i} O_{l i}}{O_{0 i}} \geq 0
$$

Notice also that if both properties occur, then the point $x_{0}$ must be the unique point where all functions $f_{i}$ are non-negative, and where all $f_{i}$ and $\left|f_{i}\right|$ are maximal.

From the symmetry of the situation, we may consider the functions

$$
g_{x}(i)=\frac{O_{x i}}{O_{0 i}}
$$

to be a UOB on the set $\{0, \ldots, n\}$ endowed with the measure $\nu(i)=O_{0 i}^{2}$.

From this we deduce that a basis has the HGP property if and only if this new basis has the GKS property, where of course the point 0 plays the role of the point $x_{0}$ in the previous paragraph. Therefore, the $g_{x}(i)$ are maximal at $i=0$, and moreover $0_{i 0}$ is minimal at $i=0$. This means that, for the HGP property also, one has

Proposition 2.4. If the $U O B \mathcal{F}$ has the HGP property at point $x_{0}$, then

$$
\forall i, x, \quad\left|f_{i}(x)\right| \leq f_{i}\left(x_{0}\right) ; \quad \forall x, \mu(x) \geq \mu\left(x_{0}\right) .
$$

(Recall that we assume that $f_{i}\left(x_{0}\right)>0$.)

We may reformulate the HGP property in the following way, which we shall use later in a different context, since it is more tractable.

Proposition 2.5. The UOB $\mathcal{F}$ has the HGP property if and only if there is a probability kernel $k(x, y, d z)$ such that, for any $i=0, \ldots, n$

$$
\frac{f_{i}(x) f_{i}(y)}{f_{i}\left(x_{0}\right)}=\int \frac{f_{i}(z)}{f_{i}\left(x_{0}\right)} k(x, y, d z) .
$$

Proof. The proof is straightforward. If the hypergroup property holds, then the kernel

$$
k(x, y, d z)=\left(\sum_{i} \frac{f_{i}(x) f_{i}(y) f_{i}(z)}{f_{i}\left(x_{0}\right)}\right) \mu(d z)
$$


is a probability kernel satisfying our conditions.

On the other hand, if such a probability kernel $k(x, y, d z)$ exists, then writing

$$
k(x, y, d z)=K_{1}(x, y, z) \mu(d z)
$$

and

$$
K_{1}(x, y, z)=\sum_{i} a_{i}(x, y) f_{i}(z)
$$

one sees that

$$
a_{i}(x, y)=\frac{f_{i}(x) f_{i}(y)}{f_{i}\left(x_{0}\right)} .
$$

The link with the Markov operators is the following.

Definition 2.3. A Markov operator is just an operator $K$ which satisfies $K(1)=1$ and which preserves non-negative functions.

Given a sequence $\lambda=\left(\lambda_{i}, i=0, \ldots, n\right)$, we define the associated linear operator $K_{\lambda}$ by

$$
K_{\lambda}\left(f_{i}\right)=\lambda_{i} f_{i} .
$$

A Markov sequence (MS in short) is a sequence $\lambda=\left(\lambda_{i}\right)$ such that the associated operator $K_{\lambda}$ is a Markov operator.

Remark that for any MS $\lambda$, one has $\lambda_{0}=1$. Remark also that

$$
K_{\lambda}(f)(x)=\int f(y) k_{\lambda}(x, d y),
$$

where

$$
k_{\lambda}(x, d y)=\left(\sum_{i} \lambda_{i} f_{i}(x) f_{i}(y)\right) \mu(d y) .
$$

Therefore, the set of MS's is just the set of sequences $\lambda$ such that the matrices $k_{\lambda}(x, y)$ are Markov matrices.

The HGP property asserts that, for any $x$, the sequence

$$
\lambda(x)=\left(\frac{f_{i}(x)}{f_{i}\left(x_{0}\right)}\right)_{i=0, \ldots, n}
$$

is a MS.

It is quite standard to see that any eigenvalue $\lambda_{i}$ of a Markov operator must satisfy $\left|\lambda_{i}\right| \leq 1$. The set of Markov sequences is a convex compact set, which is stable under pointwise multiplication. Now the main interest of this property relies in the following theorem. 
Theorem 2.1. If the basis $\mathcal{F}$ has the HGP property, then the sequences

$$
\left(\lambda_{i}(x)=\frac{f_{i}(x)}{f_{i}\left(x_{0}\right)}\right)_{i}
$$

are the extremal points in the convex set of all Markov sequences.

More precisely, every Markov sequence may be written uniquely as

$$
\left(\lambda_{i}=\int \frac{f_{i}(x)}{f_{i}\left(x_{0}\right)} d \nu(x)\right)_{i}
$$

for some probability measure $\nu$ on E. Conversely, every probability measure can be associated in the same way with a Markov sequence.

The main interest of this result is that there exist numerous natural $L^{2}$ bases with the HGP property, as we shall see later.

Proof. The representation formula is straightforward. Indeed, writing $\nu(d y)=$ $k_{\lambda}\left(x_{0}, d y\right)$, one has

$$
\lambda_{i} f_{i}\left(x_{0}\right)=\int f_{i}(y) \nu(d y)
$$

which gives the representation.

From this, it is easy to see that if the sequences $\left(\lambda_{i}(x)\right)_{i}$ are Markov sequences, then they are extremal. Indeed any representation

$$
\frac{f_{i}(x)}{f_{i}\left(x_{0}\right)}=\theta \lambda_{i}^{1}+(1-\theta) \lambda_{i}^{2}
$$

with MS's $\lambda^{1}$ and $\lambda^{2}$ leads to

$$
f_{i}(x)=\int f_{i}(y) \nu(d y)
$$

with

$$
\nu(d y)=\theta K_{\lambda^{1}}\left(x_{0}, d y\right)+(1-\theta) K_{\lambda^{2}}\left(x_{0}, d y\right) .
$$

From this we deduce that for any function $f$

$$
f(x)=\int f(y) \nu(d y),
$$

and therefore $\nu=\delta_{x}$, which gives the extremality.

Remark. Remark that the representation formula is still true for any MS when the basis does not verify the HGP property. As we can always embed the convex set of Markov sequences in the $n$-dimensional affine space $H_{1}=$ $\left\{\left(\lambda_{i}\right), \lambda_{0}=1\right\}$, the latter fact means that this set is actually contained in the $n$-simplex generated by the $n+1$ points $\lambda^{x / x_{0}}=\left(f_{i}(x) / f_{i}\left(x_{0}\right)\right)_{i}$. 
Then, when the hypergroup property holds, the set of Markov sequences is a $n$-simplex and the representation of a point in this set as affine combination of extremal points is unique.

We may ask for which kind of $L^{2}$ basis on a finite space this still happens. It is quite clear that the cardinal of the set of extremal Markov sequences is finite. Indeed, the set of Markov sequences is delimited by a finite number of $(n-1)$-hyperplanes in $H_{1}$. Namely, for any pair $(x, y)$ of points in $E$, one considers the half space defined by $\left\{\left\langle\lambda, F^{x, y}\right\rangle \geq 0\right\}$, where $F^{x, y}=\left(f_{i}(x) f_{i}(y)\right)$. Then the set of Markov sequences is the intersection of all these half spaces. Therefore, every extremal point lies in the finite set $E_{1}$ of possible intersections of $n$ hyperplanes $H^{x, y}=H_{1} \cap\left\{\left\langle\lambda, F^{x, y}\right\rangle=0\right\}$. Now, consider any point $x_{0}$ such that for any index $i, f_{i}\left(x_{0}\right) \neq 0$. The point $\lambda^{x / x_{0}}=\left(f_{i}(x) / f_{i}\left(x_{0}\right)\right)_{i}$ belongs to $H^{x_{0}, y}$ for any $y \neq x$, thanks to the orthogonality relations of the basis. Therefore, those points $\lambda^{x / x_{0}}$ belong to the set $E_{1}$. When $x_{0}$ is fixed and $x$ varies in $E$, those points describe a simplex $S_{x_{0}}$ for which we know that every Markov sequence belongs to it. The hypergroup property holds at some point $x_{0}$ exactly when no other point in $E_{1}$ lie in the interior of $S_{x_{0}}$.

On three points, one may check directly that the hypergroup property holds at some point $x_{0}$ exactly when the set of Markov sequences is a simplex (that means no other simplex is possible than the simplices $S_{x}, x \in E$ ). We may wonder if this situation is general, that is if the hypergroup property is equivalent to the fact that the set of Markov sequences is a simplex.

\subsection{Markov operators as convolutions}

When the hypergroup property holds, we may introduce a convolution on the space of measures.

Indeed, consider the kernel

$$
k(x, y, z)=\sum_{i} \frac{f_{i}(x) f_{i}(y) f_{i}(z)}{f_{i}\left(x_{0}\right)} .
$$

We observe that, for any $(x, y)$

$$
\int k(x, y, z) \mu(d z)=1
$$

and therefore the measures

$$
\mu_{x, y}(d z)=k(x, y, z) \mu(d z)
$$

are probability measures.

We may decide that the convolution is defined from this kernel by

$$
\delta_{x} * \delta_{y}=\mu_{x, y},
$$

and extending it to any measure by bilinearity. 
Moreover, we extend the convolution to functions by identifying a function $f$ with the measure $f d \mu$. This gives

$$
f * g(z)=\int f(x) g(y) k(x, y, z) d \mu(x) d \mu(y) .
$$

Observe that

$$
f_{i} * f_{j}=\delta_{i j} \frac{f_{i}}{f_{i}\left(x_{0}\right)},
$$

and that this property again completely determines the convolution.

It is easy to verify that this convolution is commutative and that $\delta_{x_{0}} * \nu=\nu$ for any $\nu$. Moreover, if an operator $K$ satisfies $K\left(f_{i}\right)=\lambda_{i} f_{i}$, then

$$
K(f * g)=K(f) * g=f * K(g),
$$

as may be verified directly when $f=f_{i}$ and $g=f_{j}$ using (2).

On the other hand, if $\nu$ is a probability measure, then the operator $K_{\nu}(f)=$ $f * \nu$ is a Markov operator which satisfies

$$
K_{\nu}\left(f_{i}\right)=\lambda_{i} f_{i},
$$

with

$$
\lambda_{i}=\frac{\int f_{i} d \nu}{f_{i}\left(x_{0}\right)} .
$$

This is straightforward using (2) if we write $\nu(d x)=h(x) \mu(d x)$ and the decomposition of $h$ along the basis $\mathcal{F}$.

Therefore, if $K$ is a Markov operator, then we have

$$
K(f)=K\left(f * \delta_{x_{0}}\right)=f * K\left(\delta_{x_{0}}\right) .
$$

This representation is exactly the representation of Markov sequences, with $\nu=K\left(\delta_{x_{0}}\right)$, and every Markov operator $K_{\lambda}$ may be defined from $K_{\lambda}(f)=$ $f * \nu$, for some probability measure $\nu$.

\subsection{The case of finite groups}

Many natural examples of finite sets endowed with a probability measure and a UOB which satisfies both GKS and HGP properties come from finite groups.

Since perhaps not every reader of these notes is familiar with this setting, let us summarize briefly the basic elements of the analysis on groups. We refer to [22] or [31] for more details.

Given a finite group $G$, one may consider linear representations $\rho: G \mapsto$ $U(V)$, that is group homomorphisms between $G$ and some $U(V)$, for some finite dimensional Hermitian space $V$ (where $U(V)$ denotes the unitary group of $V$ ). Such a representation is irreducible if there is no non-trivial proper subspace of $V$ which is invariant under $\rho(G)$. Any representation may be split 
into a sum of irreducible representations, acting on orthogonal subspaces of $V$. Two representations $\left(\rho_{1}, V_{1}\right)$ and $\left(\rho_{2}, V_{2}\right)$ are equivalent if there exists a linear unitary isomorphism $h: V_{1} \mapsto V_{2}$ such that $\rho_{1}(g)=h^{-1} \rho_{2}(g) h$ for any $g \in G$. There are only a finite number of non-equivalent irreducible representations, that we denote $\left(\rho_{i}, V_{i}\right), i \in I=\{0, \ldots, n\}$.

Let $\hat{G}$ the set of the equivalence classes of $G$ under the conjugacy relation ( $g_{1}$ is conjugate to $g_{2}$ means $g_{2}=g^{-1} g_{1} g$ for some $g \in G$ ). We endow $\hat{G}$ with the probability $\nu$ which is the image measure of the uniform measure on $G$, which means that the measure of any class is proportional to the number of points in this class. A function on $G$ which is constant on conjugacy classes (that we call a class function) can be seen as a function on $\hat{G}$. It is just a function which is stable under conjugacy.

For any irreducible representation $\left(\rho_{i}, V_{i}\right)$, let us define the function $\chi_{i}$ on $G$ by $\chi_{i}(g)=\operatorname{trace}\left(\rho_{i}(g)\right)$. This is a class function, that is to say constant on any conjugacy class. The function $\chi_{i}$ is called the character of the representation. By convention, we take $\chi_{0}=1$, that is the trace of the constant representation into the space $V=\{\mathbb{C}\}$.

Proposition 2.6. The set $\left\{\chi_{i}, i \in I\right\}$ is a (complex) UOB for $(\hat{G}, \nu)$. Moreover, it has the GKS and HGP properties.

Proof. We shall not enter in the details here. We refer to any introduction book on the representation theory of finite groups for the first fact. We shall detail a bit more the HGP and GKS properties, which are perhaps less standard.

For the GKS property, for any pair of irreducible representations $\left(\rho_{i}, V_{i}\right)$ and $\left(\rho_{j}, V_{j}\right)$, one may consider the representation $\rho_{i} \otimes \rho_{j}$ in the tensor product $V_{i} \otimes V_{j}$. If we split this representation into irreducible representations and take the trace, and if we notice that trace $\left(\rho_{1} \otimes \rho_{2}\right)=\operatorname{trace}\left(\rho_{1}\right) \operatorname{trace}\left(\rho_{2}\right)$, then we get that

$$
\chi_{i} \chi_{j}=\sum_{k} m_{i j k} \chi_{k},
$$

where $m_{i j k}$ is the number of times that the representation $\rho_{k}$ appears in this decomposition. Here we may see that not only the multiplication coefficients are non-negative, but they are integers.

We shall see next that this basis has the HGP property at the point $x_{0}=e$ (which forms a conjugacy class by itself). For that, we require a bit more material.

First define the convolution on the group $G$ itself by

$$
\phi * \psi(g)=\frac{1}{|G|} \sum_{g^{\prime} \in G} \phi\left(g g^{-1}\right) \psi\left(g^{\prime}\right) .
$$

The Fourier transform is defined on the set $I$ of irreducible representation as

$$
\hat{\phi}(i)=\sum_{g \in G} \phi(g) \rho_{i}(g) .
$$


(It takes values in the set of linear operators on $V_{i}$.)

One has an inversion formula

$$
\phi(g)=\frac{1}{|G|} \sum_{i} d_{i} \operatorname{trace}\left(\rho_{i}\left(g^{-1}\right) \hat{\phi}(i)\right),
$$

where $d_{i}$ is the dimension of $V_{i}$ (the degree of the representation).

One has

$$
(\phi * \psi)^{\wedge}=\hat{\phi} \hat{\psi}
$$

and

$$
\hat{\chi}_{j}(i)=\delta_{i j} \frac{|G|}{d_{i}} .
$$

Now, the convolution of two class functions is again a class function, as seen directly from the definition.

We want to show that this convolution is exactly the convolution that we defined in the previous section from the HGP property, that is

$$
\chi_{i} * \chi_{j}=\delta_{i j} \frac{\chi_{i} \cdot}{\chi_{i}(e)} .
$$

For that, we look at the Fourier transform and the result is straightforward, since $\chi_{i}(e)=d_{i}$.

This convolution is then the convolution defined from the $\chi_{i}$, and we have

$$
\delta_{x} * \delta_{y}=k(x, y, z) d \mu(z),
$$

where

$$
k(x, y, z)=\sum_{i} \frac{\chi_{i}(x) \chi_{i}(y) \chi_{i}(z)}{\chi_{i}(e)} .
$$

Since by construction in this case the convolution of two probability measures is a probability measure, the kernel $k(x, y, z)$ is non-negative, which proves the HGP property.

Observe that here the kernel $k(x, y, z)$ has a simple interpretation. Given 3 classes $(x, y, z)$, then

$$
k(x, y, z)=\frac{|G|}{|x||y|} m(x, y, z),
$$

where $m(x, y, z)$ is, for any point $g \in z$, the number of ways of writing $g=g_{1} g_{2}$ with $g_{1} \in x$ and $g_{2} \in y$, this number being independent of the choice of $g \in z$.

If we want to stick to real bases as we did before (and as we shall do in the next chapters), we may restrict ourselves to real groups (that is groups where $g$ and $g^{-1}$ are always in the same class), or we may agglomerate the class of $g$ with the class of $g^{-1}$. We get a new probability space, where the functions $\Re\left(\chi_{i}\right)$ form a UOB which again satisfies the GKS and HGP properties. 
It is certainly worth noticing that, unlike the convolution on $G$ itself, the convolution on $\hat{G}$ is always commutative.

Observe that taking the group $\mathbb{Z} / n \mathbb{Z}$, one gets a complex GKS and HGP UOB on the set of finite points with the uniform measure (with $f_{l}(x)=$ $\exp (2 i \pi l x))$, and that the unique real case where the measure is uniform and is GKS (the hypercube) is nothing else that the group $(\mathbb{Z} / 2 \mathbb{Z})^{n}$.

Remark. Unlike what happens for finite groups, it is not true in general that a basis $\mathcal{F}$ which has the GKS property has the dual property HGP. This is the case on two points spaces, since any orthogonal GKS matrix is symmetric (cf Page 10). If we look at the sets with three points, one may construct examples of an orthogonal matrix having the GKS property without the HGP property (and conversely, of course). In fact, consider an orthogonal matrix $\left(O_{i j}\right), 0 \leq i, j \leq 2$, with positive first row and columns. $O_{00}, O_{01}$ and $0_{10}$ determine entirely the first rows and columns, and then it is easy to see that there are only 2 orthogonal matrices with given $O_{00}, O_{01}, O_{10}$. Then, it is not hard (using a computer algebra program) to produce orthogonal matrices which have the GKS and not the HGP property, or which have neither, or both.

\subsection{On the GKS inequalities}

We conclude this section with some remarks on the correlation inequalities in statistical mechanics.

In this context, one is interested in the space of configurations of some system. We have a set of positions $i \in K, K$ being a finite set, and at each point $i \in K$ there is some random variable $x_{i}$ with values in $E$, where $E$ is some finite set, endowed with a probability measure $\mu$. One is then interested in the set $E^{K}$ of configurations, which is equipped with a measure $\mu_{H}$, where

$$
\mu_{H}(d x)=\exp (H(x)) \frac{\mu_{0}(d x)}{Z_{H}}
$$

where $\mu_{0}$ is the product measure $\mu^{\otimes K}$ on $E^{K}, H$ is some function on $E^{K}$ (the Hamiltonian), and $Z_{H}$ is the normalizing constant.

One of the basic example of spin systems is when $E=\{-1,1\}$, and $H=$ $\sum_{A} c_{A} \omega_{A}$, where the functions $\omega_{A}$ are the canonical GKS basis on $\{-1,1\}^{K}$ described before.

To study such systems (and more precisely their asymptotics when $K$ enlarges), one uses some structural inequalities. We present here two fundamental such inequalities, known as GKS inequalities, from Griffiths [27], Kelly and Sherman [32]. The GKS property for a basis has been introduced in [8], in an attempt to generalize the GKS inequality to a more general context.

The classical GKS inequalities are settled in the context of $(\mathbb{Z} / 2 \mathbb{Z})^{K}$. As before, we say that $F$ is a GKS function if $F=\sum_{A \subset K} f_{A} \omega_{A}$, where $\forall A$, $f_{A} \geq 0$.

Then we have 
Proposition 2.7. 1. (GKS1 inequality). Assume that $F$ and $H$ are GKS. Then

$$
\int F d \mu_{H} \geq 0 .
$$

2. (GKS2 inequality). Assume that $F, G$ and $H$ are $G K S$ functions. Then

$$
\int F G d \mu_{H} \geq \int F d \mu_{H} \int G d \mu_{H}
$$

The main advantage of the GKS and HGP properties is that they are stable under tensorization. That is, if one considers two sets $\left(E_{i}, \mu_{i}\right)$ with UOB bases $\mathcal{F}_{i}(i=1,2)$, then, on the set $\left(E_{1} \times E_{2}, \mu_{1} \otimes \mu_{2}\right)$ one has a natural UOB basis $\mathcal{F}_{1} \otimes \mathcal{F}_{2}=\left(f_{i} \otimes f_{j}\right)$. Then, if both $\mathcal{F}_{i}$ are GKS or HGP, the same is true for $\mathcal{F}_{1} \otimes \mathcal{F}_{2}$. This is straightforward from the definitions.

This allows us to consider a set $(E, \mu)$ with a given GKS basis $\mathcal{F}$, and then the basis $\mathcal{F}^{\otimes K}$ on $E^{K}$ is again GKS.

One has the following ([8])

Proposition 2.8. If $(E, \mu)$ has a $U O B \mathcal{F}$ which is $G K S$, then the GKS1 inequality is true.

Proof. The previous statement just means that if we define a GKS function $F$ as a function which may be written as $F=\sum_{i} F_{i} f_{i}$, where $\left(f_{i}\right)$ are the elements of $\mathcal{F}$ and $\forall i, F_{i} \geq 0$, then if $F$ and $G$ are GKS functions, one has

$$
\int F d \mu_{H} \geq 0
$$

The statement is straightforward, $\operatorname{since} \exp (H)$ is again GKS, being the sum of a series with non-negative coefficients, and so $F \exp (H)$ is itself GKS. Since any GKS function has a non-negative integral, the conclusion follows.

The GKS2 inequality is much harder. It has only be obtained in some restricted settings, like products of abelian groups, and when the basis comes from $G^{N}$ for $G$ elementary groups like dihedral groups, and some for other few groups. Nevertheless, in any example, one has both the GKS and the HGP property.

There is no example of a GKS basis where the GKS2 inequality is not satisfied. But we may restrict ourselves to a simpler setting.

Here is one conjecture that we had been unable to prove, and which motivated most of the material of this section:

Conjecture. If the $U O B \mathcal{F}$ has the $G K S$ and the HGP property, then the GKS2 inequality is true. 


\section{The hypergroup property in the infinite setting}

In the first section, we described the hypergroup property in the context of finite setS. In what follows, we consider a general probability space $(E, \mathcal{E}, \mu)$, together with a $L^{2}$ basis $\mathcal{F}=\left\{1=f_{0}, f_{1}, \ldots, f_{n}, \ldots\right)$. Very soon, we shall restrict ourselves to the case of a topological space (in fact an interval in the basic examples of Sections 4.3 and 5 ), where the functions $f_{i}$ of the basis will be continuous bounded functions. But some general properties may be stated in a more general context.

\subsection{Markov sequences associated with a UOB}

Let $(E, \mathcal{E}, \mu)$ a general probability space. In this subsection, we shall ask $(E, \mathcal{E})$ to be at least a "nice" measurable space in the context of measure theory. For us, it shall be enough to suppose that $E$ is a separable complete metric space (a polish space) and that $\mathcal{E}$ is the $\sigma$-algebra of its $\sigma$-field. Then $L^{2}(\mu)$ is separable.

We suppose that some $L^{2}(\mu)$ orthonormal basis $\mathcal{F}=\left(f_{0}, \ldots, f_{n}, \ldots\right)$ is given, with $f_{0}=1$. In what follows, we shall assume that this is a basis of the real Hilbert space $L^{2}(\mu)$, although we may as well assume that the functions $f_{n}$ may have complex values and be a basis of the complex Hilbert space. Such a unitary basis $\mathcal{F}$ will be called a Unitary Orthonormal Basis (UOB) associated with the measure $\mu$.

We are interested in bounded linear operators $K$ on $L^{2}(\mu)$, for which the functions $f_{n}$ are eigenvectors. They are uniquely determined by

$$
K\left(f_{n}\right)=\lambda_{n} f_{n}
$$

for some bounded sequence $\left(\lambda_{n}\right)$. The central question we address here is to determine for which sequences $\left(\lambda_{n}\right)$ one has

$$
K(f)(x)=\int_{E} f(y) k(x, d y),
$$

for some Markov kernel $k(x, d y)$ of probability measures on $E$.

As before, we shall call such a sequence $\left(\lambda_{n}\right)$ a Markov Sequence (MS in short) associated with the UOB $\mathcal{F}$. We shall say that the kernel $k$ (or rather $K$ with a slight abuse of notation) is associated with the MS $\left(\lambda_{n}\right)$.

In general, this is not an easy question, but as before the hypergroup property of the basis will be a way of describing all Markov sequences.

Let us start with some basic remarks.

First, for any Markov operator, $K\left(f_{0}\right)=f_{0}$, since $f_{0}$ is the constant function, and therefore $\lambda_{0}=1$.

Also, any such Markov operator is symmetric in $L^{2}(\mu)$, since we already know its spectral decomposition which is discrete and given by the basis $\mathcal{F}$. That means that for any pair $(f, g)$ of functions in $L^{2}(\mu)$, one has 


$$
\int K(f)(x) g(x) \mu(d x)=\int f(x) K(g)(x) \mu(d x) .
$$

Therefore, the measure $K(x, d y) \mu(d x)$ is symmetric in $(x, y)$.

But any Markov operator is a contraction in $L^{\infty}(\mu)$, and any symmetric Markov kernel is a contraction in $L^{1}(\mu)$, since, for any $f \in L^{2}(\mu)$,

$$
\int|K(f)| d \mu \leq \int K(|f|) d \mu=\int|f| K\left(f_{0}\right) d \mu=\int|f| d \mu .
$$

Therefore, by interpolation, $K$ is a contraction in $L^{p}(\mu)$ for any $p \geq 1$, and in particular in $L^{2}$.

We deduce from that that any MS $\left(\lambda_{n}\right)$ satisfies

$$
\forall n,\left|\lambda_{n}\right| \leq 1 \text {. }
$$

Also, if $\left(\lambda_{n}\right)$ and $\left(\mu_{n}\right)$ are MS's, with associated kernels $K$ and $K_{1}$, for any $\theta \in[0,1],\left(\theta \lambda_{n}+(1-\theta) \mu_{n}\right)$ is a MS, associated with the kernel $\theta K+(1-\theta) K_{1}$.

Therefore, the set of Markov Sequences is convex, and compact (for the product topology on $\mathbb{R}^{\mathbb{N}}$ ). This shows that describing all Markov sequences amounts to describe the extremal points of this convex set.

Notice also that the set of all Markov sequences is stable under pointwise multiplication, which corresponds to the composition of operators. In other words, if $\Theta$ is the set of extremal points in the compact set of Markov sequences, and if $\left(\lambda_{i}(\theta)\right)$ is the MS associated with the point $\theta \in \Theta$, then one has

$$
\lambda_{i}(\theta) \lambda_{i}\left(\theta^{\prime}\right)=\int_{\Theta} \lambda_{i}\left(\theta_{1}\right) R\left(\theta, \theta^{\prime}, d \theta_{1}\right),
$$

for some probability kernel $R\left(\theta, \theta^{\prime}, d \theta_{1}\right)$ on the space $\Theta$.

To determine that an operator $K$ is a Markov operator starting from its spectral decomposition, we shall need the following proposition.

Proposition 3.1. A bounded symmetric operator $K$ on $L^{2}(\mu)$ is a Markov operator if and only if

$$
K\left(f_{0}\right)=f_{0}, f \geq 0 \Longrightarrow K(f) \geq 0 .
$$

Proof. This is where we need the fact that the measure space $(E, \mathcal{E}, \mu)$ is a nice space. The conditions on $K$ are obviously necessary. To see the reverse, we apply the bi-measure theorem ([21], Page 129). We consider the map

$$
\mathcal{E} \times \mathcal{E} \mapsto[0,1]:(A, B) \mapsto \int \mathbb{1}_{A} K\left(\mathbb{1}_{B}\right) d \mu(x) .
$$

For any fixed $B$, this is a measure in $A$, and by symmetry, it is also a measure in $B$. Since our spaces are polish spaces, theses measures are tight, and therefore we may extend this operation into a measure $\mu_{K}(d x, d y)$ on the $\sigma$-algebra $\mathcal{E} \times \mathcal{E}$. The measure is symmetric, and any of its marginal is $\mu$. 
Then, we apply the measure decomposition theorem to write

$$
\mu_{K}(d x, d y)=K(x, d y) \mu(d y) .
$$

The kernel $K(x, d y)$ is exactly the kernel we are looking for.

\subsection{The hypergroup property}

The GKS property is relatively easy to state in a general context, as soon as the functions $f_{i}$ of the basis are in $L^{3}(\mu)$, since then we may just ask that

$$
\forall i, j, k, \quad \int_{E} f_{i} f_{i} f_{k} d \mu \geq 0 .
$$

But for the dual hypergroup property, one has to be a bit more cautious. In general, functions in $L^{2}$ are defined up to a set of $\mu$-measure 0 . Therefore, the meaning of $f_{i}(x) / f_{i}\left(x_{0}\right)$ is not so clear. In order to avoid difficulties, and since this shall correspond to the examples we are going to describe below, we restrict ourselves to the following setting: $E$ is a compact separable Hausdorff space, and the functions $f_{i}$ are continuous on $E$.

We may then set the following definition.

Definition 3.1. We shall say that the $U O B \mathcal{F}$ has the hypergroup property (HGP in short) at some point $x_{0} \in E$, if, for any $x \in E$, the operator defined on $\mathcal{F}$ by

$$
K_{x}\left(f_{i}\right)=\frac{f_{i}(x)}{f_{i}\left(x_{0}\right)} f_{i}
$$

is a Markov operator.

In other words, we require the sequences $\left(f_{i}(x) / f_{i}\left(x_{0}\right)\right)_{i}$ to be Markov sequences.

Observe that this implies that $\left|f_{i}\right|$ is maximal at $x_{0}$, since the eigenvalues of a Markov operator must be bounded by 1 . Since the functions $f_{i}$ are normalized in $L^{2}(\mu)$, then for any $i \in \mathbb{N}$, one has $\left|f_{i}\left(x_{0}\right)\right| \geq 1$.

As before, this definition is equivalent to the following

Proposition 3.2. The UOB $\mathcal{F}$ has the HGP property at the point $x_{0}$ if and only if there exists a probability kernel $K(x, y, d z)$ such that, for any $i \in \mathbb{N}$

$$
\frac{f_{i}(x) f_{i}(y)}{f_{i}\left(x_{0}\right)}=\int f_{i}(z) K(x, y, d z) .
$$

Proof. We shall mainly use this in the obvious way: if there is a probability kernel $K(x, y, d z)$ satisfying the hypothesis of the proposition, then the HGP property holds. In fact, if such a probability kernel $K(x, y, d z)$ exists, for any $x \in E$, the Markov kernel $K(x, y, d z)$ defines a Markov operator with Markov sequence $\left(f_{i}(x) / f_{i}\left(x_{0}\right)\right)_{i}$. 
For the reverse, if the HGP property holds, there exists for any $x$ a Markov kernel $k_{x}(y, d z)$ which satisfies

$$
\frac{f_{i}(x) f_{i}(y)}{f_{i}\left(x_{0}\right)}=\int f_{i}(z) k_{x}(y, d z)
$$

It remains to turn this family of kernels into a two parameters kernel $K(x, y, d z)$.

Sometimes, it is easier to see the HGP property in the reverse way.

Proposition 3.3. If there exists a non-negative kernel $k(x, d y, d z)$ such that for any $i, j$

$$
\int f_{i}(y) f_{j}(z) k(x, d y, d z)=\delta_{i j} \frac{f_{i}(x)}{f_{i}\left(x_{0}\right)}
$$

then the HGP property holds at the point $x_{0}$.

Proof. Remark first that from our hypotheses, the kernel $k(x, d y, d z)$ is a probability kernel (taking $i=j=0$ in the definition).

Now, the two marginals of the kernel $k(x, d y, d z)$ are equal to $\mu$, since

$$
\int f_{i}(y) k(x, d y, d z)=\delta_{0 i}
$$

which shows that those marginals and $\mu$ give the same integral to any $f_{i}$, and therefore to any $L^{2}$ function.

We may then decompose the kernel $k(x, d y, d z)=k_{1}(x, y, d z) \mu(d y)$.

Since the functions $f_{i}$ are bounded, we may consider the bounded functions

$$
H_{i}(x, y)=\int f_{i}(z) k_{1}(x, y, d z)
$$

From the definition of $k_{1}$ and the hypothesis on $k$, it is straightforward to check that

$$
\int H_{i}(x, y) f_{k}(x) f_{l}(y) \mu(d x) \mu(d y)=\delta_{i l} \delta_{i k} \frac{1}{f_{i}\left(x_{0}\right)},
$$

and hence

$$
H_{i}(x, y)=\frac{f_{i}(x) f_{i}(y)}{f_{i}\left(x_{0}\right)} .
$$

Therefore $k_{1}$ satisfies the hypotheses of Proposition 3.2 and the proof is completed.

The representation of Markov sequences is then the same than in the previous section. 
Theorem 3.1. If the UOB $\mathcal{F}$ has the hypergroup property, then any Markov sequence has the representation

$$
\lambda_{i}=\int_{E} \frac{f_{i}(x)}{f_{i}\left(x_{0}\right)} \nu(d x)
$$

for some probability measure $\nu$ on E. Moreover, the Markov sequences

$$
\left(\frac{f_{i}(x)}{f_{i}\left(x_{0}\right)}\right)_{i}
$$

are the extremal Markov sequences.

Proof. The proof is exactly similar to the finite case (see Theorem 2.1).

Remark that the series

$$
K(x, y, z)=\sum_{i} \frac{f_{i}(x) f_{i}(y) f_{i}(z)}{f_{i}\left(x_{0}\right)}
$$

does not converge in general. We shall see in the examples developed in the next section that the formal measure $K(x, y, z) \mu(d z)$ is not absolutely continuous with respect to $\mu$, and may have Dirac masses at some points.

But we may still define a convolution structure from $f_{i} * f_{j}=\delta_{i j} f_{i} / f_{i}\left(x_{0}\right)$, which maps probability measures onto probability measures, and all Markov kernels associated with $\mathcal{F}$ would be represented as $K(f)=f * \nu$, for some probability measure on $E$. We give no details here since this will not be used in the sequel.

\section{Sturm-Liouville bases and Achour-Trimèche's theorem}

\subsection{The natural UOB associated with a measure on a compact interval}

In this section, we shall consider some natural infinite UOB coming from the spectral decomposition of Sturm-Liouville operators on a compact interval of the real line.

Let us first describe the context. Consider a probability measure $\mu(d x)=$ $\rho(x) d x$ on some compact interval $[a, b] \subset \mathbb{R}$. In what follows, we shall assume for simplicity that $\rho$ is smooth, bounded above and away from 0 on $[a, b]$. The density $\rho$ is associated with a canonical differential operator

$$
L(f)(x)=f^{\prime \prime}(x)+\frac{\rho^{\prime}}{\rho}(x) f^{\prime}(x),
$$


which is symmetric in $L^{2}(\mu)$. We shall consider here $L$ acting on functions on $[a, b]$ with derivative 0 at the boundaries $a$ and $b$ (Neumann boundary conditions).

In this context, $L$ is essentially self adjoint on the space of smooth functions with $f^{\prime}(a)=f^{\prime}(b)=0$ and there is an orthonormal basis

$$
\mathcal{F}=\left(1=f_{0}, f_{1}, \ldots, f_{n}, \ldots\right)
$$

of $L^{2}(\mu)$ which is given by eigenvectors of $L$ satisfying the boundary conditions. This means that there is an increasing sequence of real numbers

$$
0=\lambda_{0}<\lambda_{1}<\cdots<\lambda_{n}<\cdots
$$

such that

$$
L f_{i}=-\lambda_{i} f_{i}, f_{i}^{\prime}(a)=f_{i}^{\prime}(b)=0 .
$$

From the standard theory of Sturm-Liouville operators, the eigenvalues $\lambda_{i}$ are non-negative and simple. Therefore, there is for any $\lambda_{i}$ a unique solution $f_{i}$ of the previous equation, which has norm 1 in $L^{2}(\mu)$ and which satisfies $f(a)>0$. We refer to any standard text book for details (see [15] or [40] for example).

This basis shall be called the canonical UOB associated with $\mu$ on $[a, b]$.

The fact that we chose to deal with the Numen boundary conditions and not with the Dirichlet boundary conditions $(f(a)=f(b)=0)$ comes from the fact that we require the function 1 to be an eigenvector of the operator.

It will be much more convenient in what follows, essentially for notations, to extend our functions by symmetry in $a$ and $b$, (and the same for $\mu$ ). In this way we may consider that we are working on functions on the real line, which are symmetric under $x \mapsto 2 a-x$, and are $2(b-a)$-periodic.

The eigenvectors are perhaps not smooth then at the boundaries $a$ and $b$, but they are at least $C^{2}$ (since they are solutions of the equation $L f_{i}=-\lambda_{i} f_{i}$ at the boundaries).

The hypergroup property is stated at some point in $[a, b]$. In the finite case, we know at which point we may expect the hypergroup property to hold: this is a point of minimal mass. In the general case, such a reasoning does not hold, since one may choose a point with minimal density to a given reference measure, but this depends on this choice.

Here, the basis $\mathcal{F}$ is the sequence of eigenvectors of an elliptic second order differential operator $L$ symmetric in $L^{2}(\mu)$. In this setting, there is a natural distance associated with the operator $L$ (in this precise example of SturmLiouville operators, this is the natural distance on $\mathbb{R}$ ). In any example we know, the point $x_{0}$ is minimal in the following sense

$$
\lim _{r \rightarrow 0} \frac{\mu\left(B\left(x_{0}, r\right)\right)}{\mu(B(x, r))} \leq 1 .
$$

We did not try to prove this in a more general context. However, it is not clear how the properties of the operator $L$ must be reflected in the properties 
of $\mathcal{F}$ to insure for example that the maximal values of the eigenvectors are attained at the same point, and that this point is of minimal mass in the sense of (3).

\subsection{Wave equations}

In this context, one has some other interpretation of the hypergroup property.

On $D=[a, b]^{2}$, we shall consider the following differential equation

$$
L_{x} F(x, y)=L_{y} F(x, y)
$$

for a function $F$ which has Neumann boundary conditions on the boundary of $D$. We shall say that such a function is a solution of the (modified) wave equation.

We have to be careful here with the regularity assumption on the function $F(x, y)$ that we require. We shall see later that given any smooth function $f(x)$ at the level $x=x_{0}$, with Neumann boundary conditions, there is exactly one smooth function $F(x, y)$ on $D$ which is solution of Equation (4) and satisfies $F\left(x, y_{0}\right)=f(x)$.

In fact, if $f(x)=\sum_{i} a_{i} f_{i}(x)$ is the $L^{2}$ orthogonal decomposition of $f$, then

$$
F(x, y)=\sum_{i} \frac{a_{i}}{f_{i}\left(y_{0}\right)} f_{i}(x) f_{i}(y)
$$

is a formal $L^{2}$ solution of the wave equation, since

$$
L_{x} F=\sum_{i} \lambda_{i} \frac{a_{i}}{f_{i}\left(y_{0}\right)} f_{i}(x) f_{i}(y)=L_{y} F .
$$

But we do not even know (for the moment) that this solution is such that $L_{x} F$ is in $L^{2}(\mu \otimes \mu)$.

Therefore, we shall say that $F$ is a weak $L^{2}$ solution of (4) if for any smooth function $G(x, y)$ with Neumann boundary conditions on $\partial D$, one has

$$
\int\left[\left(L_{x}-L_{y}\right) G(x, y)\right] F(x, y) \mu(d x) \mu(d y)=0 .
$$

Since

$$
\int L_{x}(F) G \mu(d x) \mu(d y)=\int L_{x}(G) F \mu(d x) \mu(d y)
$$

for any pair of smooth functions $F$ and $G$ satisfying the Neumann boundary conditions, then any ordinary solution is a weak one.

Now, given any $L^{2}(\mu)$ function $f(x)$, the above construction produces a weak $L^{2}(\mu \otimes \mu)$ solution $F(x, y)$ satisfying the wave equation (4), and we claim immediately that this solution is unique. In fact, writing the function $F(x, y)$ as 


$$
F(x, y)=\sum_{i j} a_{i j} f_{i}(x) f_{j}(x),
$$

and using the fact that the eigenvalues are simple, one may check that if $F$ satisfies weakly (4), then $a_{i j}=0$ if $i \neq j$, from which we deduce our claim.

Observe moreover that $a_{00}=\int F(x, y) \mu(d x) \mu(d y)$, and that for almost every $y_{0}, F\left(x, y_{0}\right) \in L^{2}(\mu)$ and that

$$
\int F\left(x, y_{0}\right) \mu(d x)=\int F(x, y) \mu(d x) \mu(d y) .
$$

It is not clear however that if $F\left(x, y_{0}\right)$ is smooth, then $F(x, y)$ is smooth. This shall be done later at least when $y_{0}=a$ or $y_{0}=b$.

The link between solutions of the wave equation and Markov kernels is the following.

If a Markov kernel is Hilbert-Schmidt (that is if its eigenvalues $\lambda_{i}$ satisfy $\left.\sum_{i} \lambda_{i}^{2}<\infty\right)$, then it may be represented as a

$$
K(f)(x)=\int f(y) k(x, y) \mu(d y)
$$

where

$$
k(x, y)=\sum_{i} \lambda_{i} f_{i}(x) f_{i}(y) .
$$

Therefore, there is a one-to-one correspondence between Hilbert-Schmidt Markov kernels and non-negative weak $L^{2}$ solutions of the wave equation which satisfy

$$
\int F(x, y) \mu(d x) \mu(d y)=1 .
$$

We then have the following

Theorem 4.1. Assume that for any function $f(x)$ on the interval $[a, b]$ with Numen boundary conditions, there exists a unique $C^{2}$ solution $H_{f}(x, y)$ of the wave equation (4) on $[a, b]^{2}$ such that $H\left(x, y_{0}\right)=f(x)$. Then, the HGP property holds at the point $y_{0}$ for the natural $U O B$ associated with $\mu$ if and only if whenever $f \geq 0$ one has $H_{f} \geq 0$ on $I^{2}$.

In other words, the hypergroup property is equivalent to the fact that the wave equation is positivity preserving.

Proof. Assume first that the hypergroup property holds at the point $y_{0}$. Take any smooth solution $F(x, y)$ of the wave equation with $F\left(x, y_{0}\right)=f(x) \geq 0$. Then, from what we just saw, one has

$$
F(x, y)=K_{y}(f)(x),
$$

where $K_{y}$ is the Markov kernel with eigenvalues $f_{i}(y) / f_{i}\left(y_{0}\right)$. Therefore, $F(x, y)$ is everywhere non-negative. 
On the other hand, assume that any smooth solution of the wave equation which is non-negative on $\left\{y=y_{0}\right\}$ is non-negative everywhere. Consider the heat kernel

$$
p_{t}(x, z)=\sum_{i} \exp \left(-\lambda_{i} t\right) f_{i}(x) f_{i}(z)
$$

We know that it is a smooth function on $D$, which is everywhere positive. Then,

$$
F_{t, z}(x, y)=\sum_{i} \frac{\exp \left(-\lambda_{i} t\right)}{f_{i}\left(y_{0}\right)} f_{i}(z) f_{i}(x) f_{i}(y)
$$

is the unique $L^{2}$ solution of the wave equation with $F_{t, z}\left(x, y_{0}\right)=p_{t}(x, z)$.

Therefore, this function is non-negative, and this shows that for any $t>0$, the sequence

$$
\exp \left(-\lambda_{i} t\right) \frac{f_{n}(z)}{f_{n}\left(y_{0}\right)}
$$

is a Markov sequence. It remains to let $t$ go to 0 to get the result, since a limit of Markov sequences is a Markov sequence.

\subsection{Achour-Trimèche's theorem and wave equations}

In what follows, we consider the case of a symmetric interval $[-b, b]$. Then we have

Theorem 4.2 (Achour-Trimèche). Let $\rho$ be a log-concave and symmetric density on $[-b, b]$. Then, the natural UOB associated with $\mu$ has the HGP property at the point $-b$. In this case, we may as well choose $x_{0}=b$. The same is true on any interval with any log-concave increasing density $\rho$.

This result is one of the very few cases when one may produce hypergroup bases without any kind of group structure on the space $E$. We shall see in the next chapter that this property holds for Jacobi polynomials, but in this case, there are at least for the integer values of the coefficients some interpretations of the convolution which reflects the group action of some orthogonal group. There is absolutely no such interpretation in this context.

In general, Achour-Trimèche's result is stated with a density $\rho$ which vanishes on the boundary. Under the conditions usually stated in AchourTrimèche's theorem, there are then no difference between Neumann and Dirichlet boundary conditions. The series

$$
\sum_{i} \frac{f_{i}(x) f_{i}(y) f_{i}(z)}{f_{i}\left(x_{0}\right)} \mu(d z)
$$

is absolutely continuous with respect to the measure $\mu$, which is not the case here.

We chose to present this result in the case where the density $\rho$ is bounded from below because it seemed to us to be more natural. 
Apparently, the proof of Achour-Trimèche's theorem had never been published. We found a mention of it in the reference book [13] and the result is announced in [1], with no proof. Most of the ideas presented here come from Achour's thesis. The idea follows a previous result of Chebli [16], which works on $[0, \infty)$ and is somehow simpler (It corresponds to the case of a concave decreasing density).

Proof. To prove this result, we shall make use of the characterization of the hypergroup property in terms of the wave equation given in Theorem 4.1. We shall see in the next paragraph that any smooth bounded function satisfying Neumann boundary conditions on $[a, b]$ has a unique extension as a smooth solution of the wave equation (4), when $x_{0}$ is one of the boundary points (see Paragraph 4.5). (This has nothing to do with the log-concavity of the measure or with the symmetry: this is just a consequence of the fact that $\log (\rho)$ is smooth and bounded.)

We first treat the case where the density $\rho$ is log-concave symmetric.

First we make use of the symmetry assumption. Then, any eigenvector of the operator $L$ on $[-b, b]$ with Neumann boundary conditions is either even or odd, since $f_{i}(-x)$ is also an eigenvector with the same eigenvalue.

Then, any $L^{2}$ solution $F(x, y)$ of the wave equation (4), written as

$$
F(x, y)=\sum_{i} a_{i} f_{i}(x) f_{i}(y)
$$

is symmetric under the change $(x, y) \mapsto(y, x)$ and under $(x, y) \mapsto(-x,-y)$.

We want to show that if $F(x,-b) \geq 0$, then $F(x, y) \geq 0$ everywhere. For this, it is enough to show this on the domain $D_{1}=\{x+y \leq 0, x \geq y\}$.

Also, we may change $F$ into $F+$ epsilon for any epsilon $>0$, and we are thus reduced to prove that the result is true when the function $f$ on the boundary is bounded below by some positive constant.

Then, a point $M \in D_{1}$, let $\Delta_{M}$ be the triangle delimited by the lines $x+y=c$ and $x-y=c^{\prime}$ passing through $M$ and the line $y=-c$. Let $M_{-}$ and $M_{+}$be the points of this triangle which lie on the line $\{y=-b\}, M_{-}$ being the left point and $M_{+}$the right one (see Figure 1). Let $F$ be a smooth solution of the wave equation (4), and let $G(x, y)=F(x, y) \rho(x) \rho(y)$.

$$
2 G(M)=G\left(M_{-}\right)+G\left(M_{+}\right)+\int_{\left[M_{-} M\right]} G(s) a_{+}(s) d s+\int_{\left[M M_{+}\right]} G(s) a_{-}(s) d s
$$

where

$$
a_{+}(x, y)=\frac{1}{\sqrt{2}}\left(\frac{\rho^{\prime}}{\rho}(x)+\frac{\rho^{\prime}}{\rho}(y)\right), a_{-}(x, y)=\frac{1}{\sqrt{2}}\left(\frac{\rho^{\prime}}{\rho}(y)-\frac{\rho^{\prime}}{\rho}(x)\right),
$$

and the integral $\int_{\left[M_{-} M\right]} H(s) d s$ and $\int_{\left[M M_{+}\right]} H(s) d s$ denote the one dimensional integrals along the segments $\left[M_{-} M\right]$ and $\left[M M_{+}\right]$against the (euclidean) length measure on those lines. 


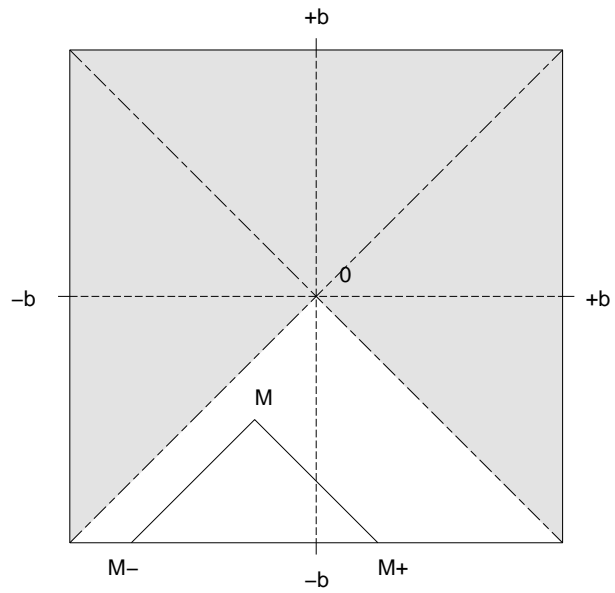

Fig. 1. Triangle $\Delta_{M}$.

This formula relies on an integration by parts formula. Even though we shall use it only on domains like $\Delta_{M}$, it is perhaps of some interest to state it in general. So we set it as a lemma. We shall not give too much details here, since a more general formula will be derived in the next paragraph.

Lemma 4.1. Let $H$ a smooth function on $D$ and $\Omega \subset D$ a domain with a piece-wise $C^{1}$ boundary $\partial \Omega$. Then

$$
\int_{\Omega}\left(L_{x}-L_{y}\right) H(x, y) \rho(x) \rho(y) d x d y=\int_{\partial \Omega} \nabla H \odot n \rho(x) \rho(y) d s,
$$

where $n=\left(n_{x}, n_{y}\right)$ denotes the exterior normal derivative of the domain and

$$
\nabla H \odot n=\partial_{x} H n_{x}-\partial_{y} H n_{y},
$$

ds designing the length measure on the boundary $\partial \Omega$.

We shall not prove this lemma. It is the analogue of the classical Stokes formula, where the elliptic operator $\Delta$ is replaced by the hyperbolic operator $L_{x}-L_{y}$, its invariant measure being $\rho(x) \rho(y) d x d y$.

One may see this as a particular case of the general integration by parts formula

$$
\int_{D} H_{1}\left(L_{x}-L_{y}\right) H_{1} \rho(x) \rho(y) d x d y=-\int \nabla H_{1} \odot \nabla H_{2} \rho(x) \rho(y) d x d y,
$$

applied with $H_{1}=\mathbb{1}_{\Delta_{M}}$.

From the previous formula, applied on $\Omega=\Delta_{M}$ for a function $F$ which is solution of the wave equation (4) and has normal derivative vanishing on the boundary $\left[M_{-}, M_{+}\right]$, one has 
$-\int_{[M-M]}\left(\partial_{x} F+\partial_{y} F\right) \rho(x) \rho(y) d s+\int_{\left[M M_{+}\right]}\left(\partial_{x} F-\partial_{y} F\right) \rho(x) \rho(y) d s=0$.

We may then perform a next integration by parts on both integrals to find

$$
\begin{aligned}
G\left(M_{-}\right)-G(M)+\int_{\left[M_{-} M\right]} G(s) a_{+}(s) d s & \\
& +G\left(M_{+}\right)-G(M)+\int_{\left[M M_{+}\right]} G(s) a_{-}(s) d s=0,
\end{aligned}
$$

which gives (5).

Under our assumptions of $\rho$, both $a_{+}$and $a_{-}$are non-negative on the subdomain $D_{1}$ : under the log-concavity assumption $a_{-}$is non-negative on $\{y \leq x\}$, and $a(x)+a(y)=a(y)-a(-x) \geq 0$ if $x+y \leq 0$.

Now, consider the smallest $y \in(-b, 0)$ such that there exists some point in $(x, y) \in D_{1}$ with $G(x, y)=0$. On this point, we have

$$
2 G(M)=0 \geq G\left(M_{-}\right)+G\left(M_{+}\right),
$$

which gives a contradiction.

For the case where the density is log-concave increasing, we may use the same argument on the domain $\{x \geq y\}$, since we still have the solution of the wave equation symmetric under the change $(x, y) \mapsto(y, x)$. Then we extend $F$ by symmetry around the axes $x=-b$ and $x=b$, and then by periodicity, into a function defined on $\mathbb{R} \times[-b, b]$. The same argument of integration by parts remains valid, and, by means of the symmetrization, the domain of integration $\left(M m_{+} M_{-}\right)$that should be used is replaced by the same triangle $\Delta_{M}$ as before, as shown in Figure 2. Then we use the fact that the function $a$ is decreasing and non-negative.

Notice that the second case (when $a$ is non-negative) may be reduced to the first one if we extend $\rho$ by symmetry around $b$, into a log-concave function on the interval $[-b, 3 b]$, symmetric around the point $x=b$. (The function $\rho$ may not be $C^{2}$ at the point $x=b$, but this causes no problem). Then one has to apply the previous result on symmetric functions on the interval $[-b, 3 b]$.

\subsection{Other representations of the solutions of the wave equation}

In this paragraph, we shall consider an operator $L(f)=f^{\prime \prime}+a(x) f^{\prime}$ in $I=[0,1]$, with Neumann boundary conditions, and look at different representations of the solutions of the wave equation (4).

We shall consider the probability measure $\rho(x) d x$ in $I$ which satisfies $\rho^{\prime} / \rho=a$.

Let $F(x, y)$ be a solution of the wave equation $\left(L_{x}-L_{y}\right) F=0$ on $I^{2}$ with Neumann boundary conditions. As before, it is easier to extend $F$ to 


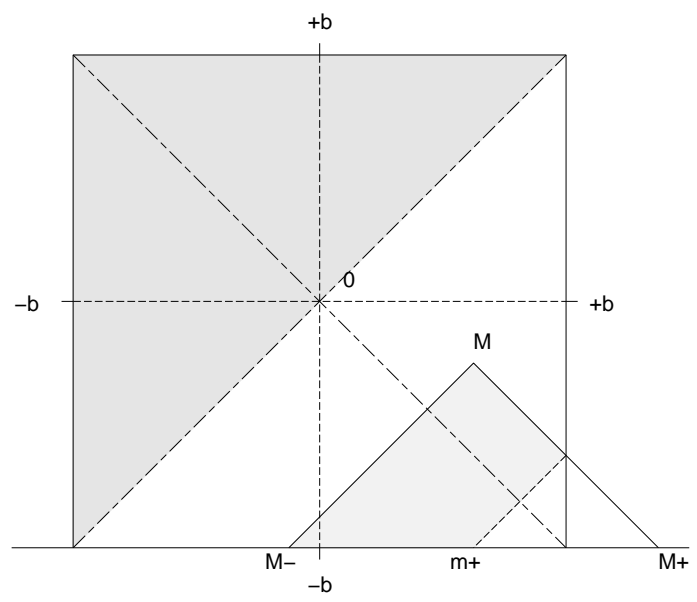

Fig. 2. Triangle $\Delta_{M}$ and $\left(M m_{+} M_{-}\right)$.

$\mathbb{R}^{2}$ by imposing symmetry conditions at the boundaries $x \in \mathbb{Z}$ or $y \in \mathbb{Z}$, and to extend $a$ by imposing antisymmetry conditions on these lines (or if one prefers, symmetry conditions on $\rho$ ).

We have seen before that such an equation has an integral representation (5). Our first task shall be to change it into a new one.

As before, for $M=(X, Y)$ with $y>0$, we denote by $\Delta_{M}$ the triangle delimited by the lines $\{x+y=X+Y\},\{y-x=Y-X\}$ and $\{y=0\} . M_{+}$ and $M_{-}$denote the edges of this triangle which lie on the line $\{y=0\}$.

For $S \in \Delta_{M}$, we denote by $S^{-M}$ the unique point $U$ on the interval $\left[M_{-}, M\right]$ such that $S \in\left[U, U_{+}\right]$and $S^{+M}$ the unique point $U$ on $\left[M, M_{+}\right]$such that $S \in\left[U_{-} U\right]$ (see Figure 3).

Recall that

$$
a_{+}(x, y)=\frac{1}{\sqrt{2}}(a(x)+a(y)), a_{-}(x, y)=\frac{1}{\sqrt{2}}(a(y)-a(x)),
$$

and let $R(x, y)=\rho(x) \rho(y)$.

Proposition 4.1. If a continuous function $G$ satisfies (5), then the function

$$
H(x, y)=\frac{G(x, y)}{\sqrt{R(x, y)}}
$$

satisfies

$$
\begin{aligned}
2 H(M)=H\left(M_{-}\right) & +H\left(M_{+}\right) \\
& +\int_{\left[M_{-}, M_{+}\right]} H(S) a_{0}(M, S) d S+\int_{\Delta_{M}} H(S) a(M, S) d S,
\end{aligned}
$$


where

$$
a(M, S)=\frac{1}{2} \sqrt{R(S)}\left(\frac{a_{+}\left(S^{-M}\right)}{\sqrt{R\left(S^{-M}\right)}} a_{-}(S)+\frac{a_{-}\left(S^{+M}\right)}{\sqrt{R\left(S^{+M}\right)}} a_{+}(S)\right),
$$

and

$$
a_{0}(M, S)=\frac{1}{2 \sqrt{2}} \sqrt{R(S)}\left(\frac{a_{+}\left(S^{-M}\right)}{\sqrt{R\left(S^{-M}\right)}}+\frac{a_{-}\left(S^{+M}\right)}{\sqrt{R\left(S^{+M}\right)}}\right) .
$$

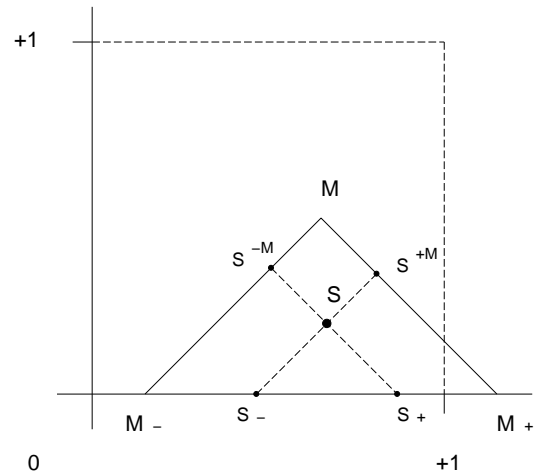

Fig. 3. $S, S_{+}, S_{-}, S^{+M}$ et $S^{-M}$.

Proof. We first start by considering the function

$$
\theta_{M}(S)=\sqrt{\frac{R(M)}{R(S)}},
$$

and we notice that, for $S \in\left[M_{-}, M\right]$,

$$
\theta_{M}(S)=1+\frac{1}{2} \int_{[S, M]} \theta_{M}(U) a_{+}(U) d U,
$$

and similarly that, for $S \in\left[M, M_{+}\right]$,

$$
\theta_{M}(S)=1+\frac{1}{2} \int_{[M, S]} \theta_{M}(U) a_{-}(U) d U
$$

Then starting from Equation (5), we replace the term 


$$
\int_{\left[M_{-} M\right]} G(S) a_{+}(S) d S
$$

by

$$
\int_{\left[M_{-} M\right]}\left(1-\theta_{M}(S)\right) G(S) a_{+}(S) d S+\int_{\left[M_{-} M\right]} \theta_{M}(S) G(S) a_{+}(S) d S .
$$

In the last integral, replace $G(S)$ by

$$
\frac{1}{2}\left(G\left(M_{-}\right)+G\left(S_{+}\right)+\int_{\left[M_{-} S\right]} G(U) a_{+}(U) d U+\int_{\left[S S_{+}\right]} G(U) a_{-}(U) d U\right) .
$$

Then we have

$$
\begin{aligned}
\int_{S \in\left[M_{-} M\right]} \theta_{M}(S) a_{+}(S)\left(\int_{U \in\left[M_{-} S\right]} G(U) a_{+}(U) d U\right) d S= \\
\int_{\left[M_{-} M\right]} G(S) a_{+}(S)\left(\int_{U \in[S, M]} \theta_{M}(U) a_{+}(U) d U\right) d S
\end{aligned}
$$

and this last expression cancels with

$$
\int_{\left[M_{-} M\right]}\left(1-\theta_{M}(S)\right) G(S) a_{+}(S) d S .
$$

We do the same computation on the other side $\left[M, M_{+}\right]$, and we collect the results. Observe that the term

$$
\int_{\left[M_{-} M\right]} \theta_{M}(S) a_{+}(S) G\left(S_{-}\right) d S
$$

gives rise to one part of the integral

$$
\int_{\left[M_{-} M_{+}\right]} H(U) a_{0}(M, U) d U
$$

while the term

$$
\int_{\left[M_{-} M\right]} \int_{\left[S S_{+}\right]} G(U) \theta_{M}(S) a_{+}(S) a_{-}(U) d S d U
$$

produces one part of the integral

$$
\int_{\Delta_{M}} H(U) a(M, U) d U
$$


We shall see in what follows that one may find many other integral representations of the wave equation.

One is the following

Proposition 4.2. Let $F$ be a solution of the wave equation (4), and let $\psi$ be a smooth positive function on $I^{2}$ satisfying the Neumann boundary conditions. Then, if we set $G(x, y)=F(x, y) \psi(x, y) \rho(x) \rho(y)$, we have

$$
\begin{aligned}
2 G(M)=G\left(M_{+}\right) & +G\left(M_{-}\right)+\int_{\left[M_{-} M\right]} G(s) K_{+}(s) d s \\
& +\int_{\left[M M_{+}\right]} G(s) K_{-}(s) d s-\int_{\Delta_{M}} G(s) \frac{\left(L_{x}-L_{y}\right)(\psi)}{\psi} d s,
\end{aligned}
$$

where

$$
K_{+}(x, y)=\sqrt{2}\left(\partial_{x}+\partial_{y}\right) \log (\psi \sqrt{\rho(x) \rho(y)})
$$

and

$$
K_{-}(x, y)=\sqrt{2}\left(\partial_{y}-\partial_{x}\right) \log (\psi \sqrt{\rho(x) \rho(y)}) .
$$

Proof. The proof is the same as before, but we have to consider the equation satisfied by $G$ instead of the equation satisfied by $F$. It is perhaps easier to make the computations under a change of variables

$$
x=\frac{u+v}{\sqrt{2}}, y=\frac{u-v}{\sqrt{2}},
$$

in which case the operator $L_{x}-L_{y}$ becomes

$$
2 \partial_{u v}^{2}-\frac{a_{-}(u, v)}{\sqrt{2}} \partial_{u}+\frac{a_{+}(u, v)}{\sqrt{2}} \partial_{v} .
$$

We extend our functions by symmetry to the set $\{y \leq 0\}$, and then our functions become for the new variables symmetric under the symmetry $(u, v) \mapsto(v, u)$. Then the result is obtained through the integration on a square $\left\{u_{0} \leq u \leq u_{1}, u_{0} \leq v \leq u_{1}\right\}$. Since we shall not use these representations here, the details are left to the reader.

As a consequence, if we set $U \leq V$ when $U \in \Delta_{V}$, and if there exists a positive function $\psi$ satisfying the Neumann boundary conditions with $\left(L_{x}-\right.$ $\left.L_{y}\right)(\psi) \leq 0$ and such that $\sqrt{\rho(x) \rho(y)} \psi$ is increasing for this partial order, then any continuous solution of the wave equation (4) which is non-negative on $\{y=0\}$ is non-negative everywhere. In particular, if there is a solution of the wave equation which is increasing for this order, then the property holds.

If we are looking for the hypergroup property at the point 0 for the Neumann basis on $[0,1]$, a good candidate for the function $\psi$ in Proposition 4.2 seems to be 


$$
\psi(x, y)=1-\frac{f(x) f(y)}{f(1)^{2}},
$$

where $f$ is the (increasing) eigenvector associated with the first non-0 eigenvalue, provided that $|f(1)| \leq|f(0)|$, which is a necessary condition for the hypergroup property to hold at 0 . But we were unable to derive reasonable conditions on $a$ which would insure that for this particular case the function $\sqrt{\rho(x) \rho(y)} \psi$ is increasing for the partial order on $[0,1]^{2}$.

\subsection{More about the solutions of the wave equation (4)}

As we saw in the previous section, there are many integral representations of the solutions of the wave equation on $[0,1]^{2}$.

Most of them appear as

$$
F(M)=\int F(S) V_{0}(M, d S)+\int F(S) V_{1}(M, d S),
$$

where $V_{0}(M, d S)$ is a continuous family of bounded measures whose support is the interval $\left[M_{-}, M_{+}\right]$on the boundary $\{y=0\}$, and $V_{1}(M, d S)$ is a continuous family of bounded measures with support $\Delta_{M}$.

In general, those representations lead to a unique representation

$$
F(M)=\int_{\left[M_{-}, M_{+}\right]} F(S) W(M, d S),
$$

for a continuous family of bounded measures with support in $\left[M_{-}, M_{+}\right]$. The crucial point is that in some situations the measure $W(M, d S)$ may be positive even if $V_{1}(M, d S)$ is not. In the case of Achour-Trimèche's theorem however, the measure $W$ is positive only on some symmetric functions.

To understand these representations, we shall consider a more general setting.

Consider a separable compact Hausdorff space $E$, and two continuous families $V_{0}(M, d s)$ and $V_{1}(M, d s)$ on $E$ (two kernels). We shall identify such a family with the operator

$$
F \mapsto V_{i}(F)(M)=\int_{E} F(y) V_{i}(M, d S),
$$

which maps the Banach space $C(E)$ of continuous functions into itself. The identity operator corresponds to the kernel $I(M, d S)=\delta_{M}(d S)$, and the composition of kernels

$$
V \odot W(M, d S)=\int_{U} V(M, d U) W(U, d S)
$$

corresponds then to the operator composition.

We set 


$$
\|V\|=\sup _{M} \int_{E}|V(M, d S)|
$$

which is the operator norm.

Then we have

Lemma 4.2. Consider some continuous function $F \in C(E)$ satisfies

$$
\forall M \in E, F(M)=\int F(S) V_{0}(M, d S)+\int_{E} F(S) V_{1}(M, d S) .
$$

If the series

$$
\sum_{n}\left\|V_{1}^{\odot n}\right\|
$$

converges, then setting

$$
\mathcal{E}\left(V_{1}\right)=W_{1}=\sum_{n} V_{1}^{\odot n}
$$

one has

$$
F=W_{1} \odot V_{0}(F) .
$$

In particular, if $V_{0}$ is supported by some closed subset $E_{0}$ of $E$, and if the condition (7) is satisfied for $V_{1}$, then there exists a unique solution $F$ to the equation (6) given the restriction $F_{0}$ of $F$ on $E_{0}$.

Moreover, if the kernels $V_{0}$ and $V_{1}$ are non-negative and if $F_{0}$ is nonnegative, so is $F$.

Proof. The proof is straightforward and is just the classical representation of $\left(I-V_{1}\right)^{-1}$ as $\sum_{n} V_{1}^{\odot n}$. Observe moreover that if $V_{1}$ is non-negative, so is $W_{1}=\mathcal{E}\left(V_{1}\right)$, and that the representation of the solution is then given by a non-negative kernel.

In what follows, and to apply these lemmas, we shall consider the case where $E$ is a compact subset of $\mathbb{R} \times[0, \infty[$, and where, for some point $M \in E$, the measure $V_{0}(M, d S)$ is supported by $\left[M_{-}, M_{+}\right], V_{1}(M, d S)$ is supported by $\Delta_{M}$, and has a bounded density $a(M, S)$ with respect to the Lebesgue measure on the product, in which case we write

$$
F(M)=\int_{\left[M_{-}, M_{+}\right]} F(S) V_{0}(M, d S)+\int_{\Delta_{M}} F(S) a(M, S) d S
$$

Then we have

Proposition 4.3. Let $\kappa$ a uniform bound on $|a(S, U)|,(S, U) \in \Delta_{M}$ in (8). Then, on $\Delta_{M}$, we have

$$
\left\|V_{1}^{\odot n}\right\| \leq \frac{\kappa^{n}\left|\Delta_{M}\right|^{n}}{(n !)^{2}}
$$

where $\left|\Delta_{M}\right|$ denotes the area of the triangle $\Delta_{M}$. 
Proof. Recall the partial order $\left(S_{1} \leq S_{2}\right) \Longleftrightarrow S_{1} \in \Delta_{S_{2}}$.

Then one has

$$
V_{1}^{\odot n}(M, d S)=\mathbb{1}_{\Delta_{M}}(S) a_{n}(M, S) d S,
$$

where

$$
\begin{aligned}
& a_{n}(M, S)= \\
& \quad \int_{S \leq S_{1} \leq \ldots \leq S_{n-1} \leq M} a\left(M, S_{n-1}\right) a\left(S_{n-1}, S_{n-2}\right) \ldots a\left(S_{1}, S\right) d S_{1} \ldots d S_{n-1} .
\end{aligned}
$$

It is easy to see by induction that

$$
\left|a_{n}(M, S)\right| \leq \kappa^{n} \frac{|[S, M]|^{n}}{n !^{2}}
$$

where $|[S, M]|$ denotes the area of the rectangle $\{U \mid S \leq U \leq M\}$.

The conclusion follows easily from this estimate.

Considering the representation of the solutions of the wave equation given in Proposition 4.1, we see that any continuous solution may be represented as

$$
F(M)=\int_{\left[M_{-} M_{+}\right]} F(S) V(M, d S)
$$

where $V(M, d S)$ has two Dirac masses at the points $M_{-}$and $M_{+}$and has a bounded density on $\left(M_{-}, M_{+}\right)$. The smoothness of this density depends of course on the smoothness of the function $a$ itself (and may be analyzed through the convergence of the series that we just described). For example, if $a$ has $k$ bounded derivative, then so has the density.

We may observe the following.

Corollary 4.1. Consider a solution $F$ of Equation (6). Assume that $V_{0}$ is non-negative and supported by $E_{0} \subset E$, and that $V_{1} \geq V_{2}$, in the sense that $V_{3}=V_{1}-V_{2}$ is a non-negative kernel. Suppose that $V_{2}$ and $V_{3}$ satisfy the growth condition (7) and moreover that $\mathcal{E}\left(V_{2}\right)=W_{2}$ is non-negative.

Then, if the restriction $F_{0}$ of $F$ on $E_{0}$ is non-negative, then $F$ is nonnegative everywhere on $E$.

Proof. Once again, this is straightforward. Setting $W_{2}=\left(I-V_{2}\right)^{-1}$, one has

$$
F=W_{2} \odot V_{0}(F)+W_{2} \odot V_{3}(F),
$$

which is an equation of the same type, but with non-negative kernels.

We may apply this for example for the solutions of (8) : 
Corollary 4.2. If $V_{0}(M, d S)$ is non-negative and $a(U, S) \geq-C$, on $S \leq U \leq$ $M$, where

$$
C=\frac{\mu_{0}^{2}}{2\left|\Delta_{M}\right|}
$$

and $\mu_{0}$ is the first 0 of the Bessel $G$ function which is solution on $(0, \infty)$ of

$$
G^{\prime \prime}+\frac{G^{\prime}}{x}=-G, G(0)=1, G^{\prime}(0)=0,
$$

then any continuous solution of Equation (8) which is non-negative on the boundary $\{y=0\}$ is non-negative on $\Delta_{M}$.

Proof. It is a simple application of the previous Corollary 4.1 with $V_{2}(M, d S)=$ $-C \mathbb{1}_{\Delta_{M}}(S) d S$.

In this case, it is not hard to see that

$$
\mathcal{E}\left(V_{2}\right)(M, d S)=\mathbb{1}_{\Delta_{M}}(S) F(|[S, M]|) d S,
$$

where $|[S, M]|$ denotes the Lebesgue measure of the rectangle $[S, M]=$ $\{U \mid S \leq U \leq M\}$ and

$$
F(x)=\sum_{n} \frac{(-C x)^{n}}{(n !)^{2}} .
$$

The function $F$ is the solution of

$$
x F^{\prime \prime}+F^{\prime}=-C F, F(0)=1, F^{\prime}(0)=-C,
$$

which is related to the function $G$ through the change of variable $x=z^{2} / 4 C$. The function $G$ is non-negative on $\left[0, \mu_{0}\right)$ and this gives the result provided one observes that $|[S, M]| \leq \frac{\left|\Delta_{M}\right|}{2}$.

Remark. One may also observe that $1 / \mu_{0}^{2}$ is the fundamental eigenvalue of the Laplace operator on the unit ball of $\mathbb{R}^{2}$ with the Dirichlet boundary conditions, the function $G\left(\|x\| / \mu_{0}\right)$ being the corresponding eigenvector.

All these considerations provide many criteria on the function $a$ such that the associated Neumann basis on $I$ has the hypergroup property at the left end point of the interval. In the next section, we shall deal with Gasper's theorem, where $I=[0, \pi / 2]$ and $a(x)=\alpha \tan x-\beta \cot x$, with $\alpha \geq \beta>-1$. The reader should check that no one of these criteria may apply on this example. Achour-Trimèche's theorem shows that the hypergroup property holds for this example in the symmetric case $\alpha=\beta$, even on any symmetric (around $\pi / 4)$ subinterval of $[0, \pi / 2]$. But we do not even know for the moment if the hypergroup property holds in the general case on any symmetric subinterval of $[0, \pi / 2]$. (Yet it is true for small subintervals and also provided that the parameters $\alpha$ and $\beta$ belong to some specific domains that we shall not describe here). 


\section{The case of Jacobi polynomials: Gasper's theorem}

Gasper's theorem states the hypergroup property for the family of Jacobi polynomials. The case of Jacobi polynomials may be considered as a special case of a Sturm-Liouville basis on $[0, \pi / 2]$. In this situation, both the GKS and the HGP property hold $[24,25,26]$. Actually, it is a unique situation for orthogonal polynomials, since they are the only ones, up to a linear change of variables, for which the HGP property holds (see $[18,17,19]$ ) (under some mild extra condition on the support of the measure which represents the product formula). In the case of symmetric Jacobi polynomials (known as Gegenbauer or ultraspherical polynomials), the HGP property may be seen as a particular example of Achour-Trimèche's theorem (although in this case the measure has a density which vanishes on the boundary). But in the general case, as we already mentioned, none of the extension we gave of Achour-Trimèche's theorem covers this result. Even worse, we do not know if the HGP property holds for any symmetric subinterval of $[0, \pi / 2]$.

The Jacobi polynomials are a quite universal object, since they are basically the unique examples of a family of orthogonal polynomials which are also eigenvectors of Sturm-Liouville operators (together with their limiting cases the Hermite and Laguerre polynomials, see [36]). On the other hand, for special values of the parameters, they may be considered as eigenvectors of rank-one symmetric compact spaces (here, with our notations, it is for the parameters $(1, p),(2, p),(4, p)$ and $(p, p)$, with $p \in \mathbb{N})$. But for a wider range of parameters $(p, q \in \mathbb{N})$, they may be seen as eigenvectors of a Laplace operator on a $p+q-1$ dimensional sphere. The special case where $p=q$ is much simpler, since then one may consider a $p$-dimensional sphere.

In this section, after a short introduction on Jacobi polynomials and the statement of the hypergroup property for these polynomials, we present the simpler case of symmetric Jacobi polynomials, where the convolution structure has a nice geometric interpretation for $p \in \mathbb{N}$. This interpretation is for example described in [12]. For the dissymmetric case, although the Jacobi polynomials still have a simple geometric interpretation too when the parameters are integers, the convolution structure is far less obvious.

\subsection{Jacobi polynomials}

This polynomial family is defined for some positive parameters $p$ and $q$ as the family of orthogonal polynomials associated with the measure

$$
\mu_{p, q}(d x)=C_{p, q}(1-x)^{\frac{q-2}{2}}(1+x)^{\frac{p-2}{2}} d x
$$

on $[-1,1], C_{p, q}$ being a normalizing constant such that $\mu_{p, q}$ is a probability measure.

These polynomials are also the eigenvectors of the operator 


$$
L_{p, q} f(x)=\left(1-x^{2}\right) f^{\prime \prime}(x)-\left(q \frac{x+1}{2}+p \frac{x-1}{2}\right) f^{\prime}(x)
$$

on $[-1,1]$. If $P_{k}^{p, q}$ is the polynomial of degree $k$, one has

$$
L_{p, q} P_{k}^{p, q}=-k\left(\frac{p+q}{2}+k-1\right) P_{k}^{p, q} .
$$

Remark. These polynomials are traditionally parametrized by $\alpha=\frac{q-2}{2}$ and $\beta=\frac{p-2}{2}$ with $\alpha, \beta>-1$, from [39] or $[24,25,26]$.

If we change $x=\cos (2 \theta), \theta \in\left[0, \frac{\pi}{2}\right]$, then this operator is turned into

$$
L_{p, q} f(\theta)=\frac{1}{4}\left[f^{\prime \prime}(\theta)+((q-1) \cot (\theta)-(p-1) \tan (\theta)) f^{\prime}(\theta)\right]
$$

We see then that Jacobi polynomials is one example of a Neumann basis associated with a Sturm-Liouville operator (except that the density of the measure vanishes on the boundary points, for parameters larger than 2). We may also observe that the measure is log-concave as soon as $p$ and $q$ are in $[1, \infty)$.

When $p$ and $q$ are integers, one may see the operator $L_{p, q}$ as the action of some spherical laplacian on a quotient of the sphere.

More explicitly, we set $N=p+q$. Let us denote by $|X|$ the euclidean norm of a point $X$ in $\mathbb{R}^{N}$, and let $\mathbb{S}^{N-1}$ be the unit sphere. We consider the Laplace operator $\Delta_{\mathbb{S}}$ on the unit sphere $\mathbb{S}^{N-1}$ in $\mathbb{R}^{N}$ : this is the restriction to the sphere of the usual Laplace operator on $\mathbb{R}^{N}$ acting on function which are defined in a neighborhood of the sphere and do not depend on the radius of the point.

We parametrize $\mathbb{S}^{N-1}$ as

$$
X=\left(\sqrt{\frac{1+x}{2}} X_{1}, \sqrt{\frac{1-x}{2}} X_{2}\right)
$$

where $X_{1} \in \mathbb{S}^{p-1} X_{2} \in \mathbb{S}^{q-1}$, and $x \in[-1,1]$. The action of $\Delta_{\mathbb{S}}$ on a function which depends only on $x$ gives again a function of $x$, and we have

$$
\Delta_{\mathbb{S}}(h)(x)=4 L_{p, q}(h)(x) .
$$

We shall say that such a function on the sphere which depends only on $x$ (that is which depends only on the norm of the projection of $X$ onto $\mathbb{R}^{p}$ ) has the invariance $S O(p) \times S O(q)$, where the action of $S O(p) \times S O(q)$ is obtained by the action of the first component on $X_{1}$ and of the second on $X_{2}$.

The measure $\mu_{p, q}$ is the invariant measure for the operator $L_{p, q}$ and the uniform measure is the invariant measure for the Laplace operator on the sphere. This shows that $\mu_{p, q}$ is the image of the uniform measure on the 
sphere (normalized as to be a probability measure) under the map $X \mapsto x$ of Formula (9).

In fact, under this map $X \mapsto\left(X_{1}, X_{2}, x\right)$, it is straightforward to see that the uniform measure $\sigma_{p+q-1}$ on $\mathbb{S}^{p+q-1}$ is transformed into $\sigma_{p-1} \otimes \sigma_{q-1} \otimes \mu_{p, q}$.

Thanks to this remark, consider $N \geq p$ and look at the projection $\pi(X)$ from $\mathbb{S}^{N-1}$ onto the unit ball in $\mathbb{R}^{p}$. (That is the orthogonal projection when the sphere is imbedded into $\left.\mathbb{R}^{N}\right)$. If we set $x=2|\pi(X)|-1$ and $X_{1}=\frac{\pi(X)}{|\pi(X)|}$, we see that the image measure of $\sigma_{N-1}$ under $X \mapsto\left(x, X_{1}\right)$ is $\mu_{p, N-p} \otimes \sigma_{p-1}$. This remark shall be used in Paragraph 5.4.

\subsection{Gasper's result}

Gasper proved the following product formula which gives the HGP property for Jacobi polynomials, applying Proposition 3.2.

Theorem 5.1 (Gasper). Let $p, q>0$ and $-1<x, y<1$. Then

- we have the following product formula:

$$
\forall k, \quad \frac{P_{k}^{p, q}(x) P_{k}^{p, q}(y)}{P_{k}^{p, q}(1)}=\int P_{k}^{p, q}(z) m_{p, q}(x, y, d z)
$$

where $m_{p, q}(x, y, d z)$ is a Borel measure on $[-1 ; 1]$;

- the measure $m_{p, q}$ is positive (and then is a probability measure) if and only if

$$
(p, q) \in\{q \geq p\} \cap\{p \geq 1 \text { or } p+q \geq 4\} ;
$$

- moreover, if $q>p>1, m_{p, q}(x, y, d z)$ is absolutely continuous with respect to $\mu_{p, q}$, with density in $L^{2}\left(\mu_{p, q}\right)$, so that

$$
K_{p, q}(x, y, z)=\sum_{k} \frac{P_{k}^{p, q}(x) P_{k}^{p, q}(y) P_{k}^{p, q}(z)}{P_{k}^{p, q}(1)} \geq 0,
$$

with convergence of the sum for almost every $z$.

The original Gasper's proof (see $[25,26]$ ) consisted in the explicit computation of the sum $K_{p, q}(x, y, z)$ using formulae on special functions like Bessel's and hypergeometric functions. There had been many other proofs of this property. For example, Koornwinder derived it in [35] from the addition formula of Jacobi polynomials and he found an other proof in [34], that we discuss next.

Here we restrict ourself to prove the HGP property in the symmetric case $(p=q)$ and in the case when $q>p>1$. In the latter case, we follow a proof given by Koornwinder in [34]. However, his argument was based on an integral representation formula of the polynomials (our Lemma 5.2), whose proof, as we found in literature (see [3] together with [4]), relies on computational considerations on hypergeometric functions. In Section 5.4, we shall give a more geometric interpretation of this formula, at least when $p$ and $q$ are integers (it appears finally that the interpretation of Jacobi polynomials as harmonic functions was already known - see [14, 23, 35] - but it seems that it was not yet directly used to derive Koornwinder's representation formula). 


\subsection{The special case of ultraspherical polynomials $(p=q \geq 1)$}

In the case when $p=q$, Jacobi polynomials are called ultraspherical polynomials. In this case, there is a much simpler representation of $L_{p, p}$ when $p \in \mathbb{N}^{*}$ as the action of the Laplace operator on the sphere $\mathbb{S}^{p}$ (and not on $\mathbb{S}^{2 p-1}$ as before).

When $p$ is a positive integer, then the hypergroup property has a simple geometric interpretation, and thus the property is quite easy to establish. This easily extends to the case when $p \notin \mathbb{N}$, by a simple extension of the formulae. This is what we are going to see in this paragraph.

Consider a smooth function $F: \mathbb{S}^{p} \mapsto \mathbb{R}$ which depends only on the first coordinate. To fix the ideas, let $F(X)=f\left(X \cdot e_{1}\right)$, where $e_{1}$ is the first unit vector in $\mathbb{R}^{p+1}$, and $Y \cdot X$ denotes the standard scalar product in $\mathbb{R}^{p+1}$. Then, if $\Delta_{\mathbb{S} p}$ is the Laplace operator on $\mathbb{S}^{p}$, we have

$$
\Delta_{\mathbb{S} p}=L_{p, p}(f)\left(X \cdot e_{1}\right) .
$$

As before, the image measure of the uniform measure on the sphere through the map $X \mapsto x=X \cdot e_{1}$ is the invariant measure for $L_{p, p}$, that is $\mu_{p, p}$. Moreover, we may parametrize $\mathbb{S}^{p *}=\mathbb{S}^{p} \backslash\left\{e_{1},-e_{1}\right\}$ by

$$
X=\left(x, \sqrt{1-x^{2}} X_{1}\right),
$$

where $x \in(-1,1)$ is the first coordinate of the point $x \in \mathbb{S}^{p} \subset \mathbb{R}^{p+1}$, and

$X_{1} \in \mathbb{S}^{p-1}$. Through this map $\mathbb{S}^{p *} \mapsto(-1,1) \times \mathbb{S}^{p-1}$, the image measure of $\sigma_{p}$ is $\mu_{p, p} \otimes \sigma_{p-1}$.

From that, we see that if $P_{k}^{p, p}$ is the $k$-th ultraspherical polynomial, then $P_{k}^{p, p}\left(X . e_{1}\right)$ is an eigenvector of $\Delta_{\mathbb{S} p}$, with eigenvalue $\lambda_{k}^{p}=-k(k+p-1)$.

Observe that for any point $Y$ on the sphere, $P_{k}^{p, p}(Y . X)$ is again an eigenvector on the sphere with the same eigenvalue. (This comes from the fact that the Laplace operator on the sphere is invariant under rotations.)

Now, if we take two points $Y$ and $Z$ on the sphere, $P_{k}^{p, p}(Y . X)$ and $P_{k}^{p, p}(Z . X)$ are two eigenvectors of $\Delta_{\mathbb{S} p}$, with the same eigenvalue. Let us compute their scalar product in $L^{2}\left(\mathbb{S}^{p}\right)$

$$
H(Y, Z)=\int_{\mathbb{S}^{p}} P_{k}^{p, p}(Y . X) P_{k}^{p, p}(Z . X) \sigma_{p}(d X) .
$$

Obviously, $H(Y, Z)$ is a smooth function, taking values in $[-1,1]$, and if $R$ is any rotation, $H(Y, Z)=H(R Y, R Z)$. From this we see that $H(Y, Z)=h(Y$. $Z)$, and we may write the function $h$ in terms of ultraspherical polynomials

$$
h=\sum_{r} a_{r} P_{r}^{p, p} .
$$

We have 


$$
\begin{aligned}
a_{r} & =\int h(x) P_{r}(x) \mu_{p, p}(d x)=\int_{\mathbb{S}^{p}} h(Y . Z) P_{r}^{p, p}(Y . Z) \sigma_{p}(d Z) \\
& =\int_{\mathbb{S}^{p}} \int_{\mathbb{S}^{p}} P_{r}^{p, p}(Y . Z) P_{k}^{p, p}(Y . X) P_{k}^{p, p}(Z . X) \sigma_{p}(d X) \sigma_{p}(d Z) .
\end{aligned}
$$

Using Fubini's theorem and the orthogonality of eigenvectors associated with different eigenvalues, we see that $a_{r}=0$ unless $r=k$. We therefore see that

$$
\int_{\mathbb{S}^{p}} P_{k}^{p, p}(Y . X) P_{k}^{p, p}(Z . X) \sigma_{p}(d X)=a_{k} P_{k}^{p, p}(Y . Z) .
$$

To compute $a_{k}$ we choose $Y=Z$, from which we get, if we remember that the polynomials $P_{k}^{p, p}$ have norm 1 in $L^{2}$, that

$$
a_{k} P_{k}^{p, p}(1)=1 \text {. }
$$

Now, if we rewrite this formula for $Y=e_{1}$ and through the parametrization described above in (10), then we get, for $Z=\left(z, \sqrt{1-z^{2}} Z_{1}\right)$,

$\frac{P_{k}^{p, p}(z)}{P_{k}^{p, p}(1)}=\int_{x, X_{1}} P_{k}^{p, p}(x) P_{k}^{p, p}\left(z x+\sqrt{1-z^{2}} \sqrt{1-x^{2}} X_{1} Z_{1}\right) \mu_{p, p}(d x) \sigma_{p-1}(d X)$,

while, for $k \neq l$,

$$
\int_{x, X_{1}} P_{k}^{p, p}(x) P_{l}^{p, p}\left(z x+\sqrt{1-z^{2}} \sqrt{1-x^{2}} X_{1} Z_{1}\right) \mu_{p, p}(d x) \sigma_{p-1}(d X)=0 .
$$

This may be rewritten as

$$
\int_{x, t} P_{k}^{p, p}(x) P_{l}^{p, p}\left(z x+\sqrt{1-z^{2}} \sqrt{1-x^{2}} t\right) \mu_{p, p}(d x) \mu_{p-1, p-1}(d t)=\delta_{k l} \frac{P_{k}^{p, p}(z)}{P_{k}^{p, p}(1)} .
$$

This last formula may be turned into an explicit representation

$$
\int_{x, t} P_{k}^{p, p}(x) P_{l}^{p, p}(y) k_{p}(z, d x, d y)=\delta_{k l} \frac{P_{k}^{p, p}(z)}{P_{k}^{p, p}(1)}
$$

for some probability kernel $k_{p}(z, d x, d y)$, which gives the hypergroup property thanks to Proposition 3.3.

When $p$ is not an integer, since we have an explicit representation of the kernel $k_{p}(z, d x, d y)=K_{p}(x, y, z) \mu(d x) \mu(d y)$, it is a simple verification to check that the function $K_{p}(x, y, z)$ satisfies $L_{x} K_{p}=L_{y} K_{p}$ together with

$$
\lim _{y \rightarrow 1} K_{p}(x, y, z) \mu_{p, p}(d z)=\delta_{x}(d z),
$$

which is enough to get the HGP property at the point 1.

Moreover, the convolution associated with this hypergroup structure is quite easy to understand when $p$ is an integer. 
Let us say that a probability measure $\mu$ on the sphere $\mathbb{S}^{p}$ is zonal around $X \in \mathbb{S}^{p}$ if it is invariant under any rotation $R \in S O(p+1)$ such that $R X=X$.

Given any probability measure $\mu$ on $[-1,1]$, and any $X \in \mathbb{S}^{p}$, we may lift $\mu$ into a unique probability measure $\hat{\mu}$ which is zonal around $X$ such that the image measure of $\hat{\mu}$ under the projection $\pi(Y)=Y \cdot X$ from $\mathbb{S}^{p}$ onto $[-1,1]$ is $\mu$.

Now, let us choose $e_{1} \in \mathbb{S}^{p}$, and consider two probability measures $\nu_{1}$ and $\nu_{2}$ on $[-1,1]$. We may lift $\nu_{1}$ into a probability measure $\hat{\nu}_{1}$ on $\mathbb{S}^{p}$, which is zonal around $e_{1}$. Then, we choose a random point in $\mathbb{S}^{p}$ according to $\hat{\nu}_{1}$. Then, given $X$, we consider the lift of $\nu_{2}$ which is zonal around $X$ and choose a random point $Y$ according to this measure. Then, the resulting law of $Y$ is zonal around $e_{1}$, and we project this measure into a new measure $\nu_{1} * \nu_{2}$. It is an exercise to show that this convolution is the convolution associated with the hypergroup structure in this case.

\subsection{The case of dissymmetric Jacobi polynomials $(q>p>1)$}

Although the dissymmetric Jacobi polynomials may be interpreted as eigenvectors of the Laplace operator on the sphere $\mathbb{S}^{p+q-1}$, it is far from trivial to prove the hypergroup property even in the case where $p$ and $q$ are integers. Nevertheless, the proof that we present below for completeness and which is due essentially to Koornwinder [34] has also some simple interpretation when $p$ and $q$ are integers in terms of harmonic analysis in $\mathbb{R}^{p+q}$.

Koornwinder's proof relies on two facts, given in the following Lemmas 5.1 and 5.2. In what follows, and to lighten the notations, we remove the indices $p$ and $q$ from the definitions of the polynomials $P_{k}^{p, q}$.

Lemma 5.1. (Bateman's formula) Let $b_{k, r}$ the the coefficients such that

$$
\frac{P_{k}(s)}{P_{k}(1)}=\sum_{r=0}^{k} b_{k, r}(s+1)^{r}
$$

Then

$$
\frac{P_{k}(s) P_{k}(t)}{P_{k}(1)^{2}}=\sum_{r=0}^{k} b_{k, r} \frac{(s+t)^{r}}{P_{r}(1)} P_{r}\left(\frac{1+s t}{s+t}\right) .
$$

Lemma 5.2. (Koornwinder's formula)

$$
\begin{aligned}
\frac{P_{k}(x)}{P_{k}(1)}=\int_{[-1,1]^{2}} & {\left[\frac{2(1+x)-(1-x)(1+u)}{4}\right.} \\
& \left.+\frac{\mathrm{i} \sqrt{2} \sqrt{1-x^{2}} \sqrt{1+u} v}{4}\right]^{k} \mu_{p, q-p}(d u) \mu_{p-1, p-1}(d v) .
\end{aligned}
$$


Before going further, let us show that this implies the HGP property at the point $x_{0}=1$. In fact, we shall use the characterization of the hypergroup property given by Proposition 3.2.

For that, we replace in Bateman's formula of 5.1 the representation given by Koornwinder's formula. For this, we observe that, if $(s, t) \in(0,1)^{2}$, then

$$
\left(\frac{1+s t}{s+t}\right)^{2}>1
$$

and therefore if we set $x=(1+s t) /(s+t)$, we may replace by analytic continuation $\mathrm{i} \sqrt{1-x^{2}}$ by $\sqrt{x^{2}-1}$.

Then, if we set

$$
\psi(s, t, u, v)=\frac{2(1+x)-(1-x)(1-u)+\sqrt{2} \sqrt{x^{2}-1} \sqrt{1+u} v}{4},
$$

one has

$$
\frac{P_{k}(s) P_{k}(t)}{P_{k}(1)^{2}}=\int \sum_{r=0}^{k} b_{k, r}[(s+t) \psi(s, t, u, v)]^{k} \mu_{p, q-p}(d u) \mu_{p-1, p-1}(d v) .
$$

From the definition of the coefficients $b_{k, r}$, we get then

$$
\frac{P_{k}(s) P_{k}(t)}{P_{k}(1)^{2}}=\int \frac{P_{k}((s+t) \psi(s, t, u, v)-1)}{P_{k}(1)} \mu_{p, q-p}(d u) \mu_{p-1, p-1}(d v) .
$$

If we define $m_{p, q}(s, t, d z)$ to be the image measure of $\mu_{p, q-p}(d u) \mu_{p-1, p-1}(d v)$ under the map

$$
(u, v) \mapsto(s+t) \psi(s, t, u, v)-1,
$$

one gets

$$
\frac{P_{k}(s) P_{k}(t)}{P_{k}(1)}=\int P_{k}(z) m_{p, q}(s, t, d z),
$$

which is the announced result.

Of course, one has to check that the image measure is indeed supported by $[-1,1]$, but this point is left to the reader.

We now give the proof of Lemmas 5.1 and 5.2. As it shall turn out, they rely on elementary considerations on the interpretations of the operator $L_{p, q}$. For the moment, we restrict ourselves to the case where $p$ and $q$ are positive integers, and we shall interpret those formulae in term of the Laplace operator on $\mathbb{R}^{p+q}$.

First observe that, given any function $f$ on $[-1,1]$, we may lift this function on the sphere $\mathbb{S}^{p+q-1}$ into a function which has the $S O(p) \times S O(q)$ invariance. Namely, using the parametrization of the sphere given in (9), we set

$$
F\left(\sqrt{\frac{1+x}{2}} X_{1}, \sqrt{\frac{1-x}{2}} X_{2}\right)=f(x) .
$$


Notice that in this formula,

$$
x=\left|\pi_{1} X\right|^{2}-\left|\pi_{2}(X)\right|^{2},
$$

where $\pi_{1}$ and $\pi_{2}$ are the orthogonal projections on $\mathbb{R}^{p}$ and $\mathbb{R}^{q}$ when the sphere is imbedded into $\mathbb{R}^{p+q}$. Let us call $U(f)$ such a lift of a function from $[-1,1]$ onto the sphere.

Now, if $P_{k}$ is the Jacobi polynomial of degree $k$, the corresponding function $F_{k}=U\left(P_{k}\right)$ is an eigenvector of the Laplace operator on the sphere, and therefore the restriction to the sphere of a harmonic polynomial of degree $2 k$. Therefore, if we parametrize a point $Z$ in $\mathbb{R}^{p+q}$ by $R=|Z|^{2}$ and $X=\frac{Z}{|Z|}$, we may see that the function $R^{k} F_{k}(X)$ is harmonic in $\mathbb{R}^{p+q}$.

This may be seen in another way as we may write the Laplace operator in those coordinates

$$
\Delta=\frac{\partial^{2}}{\partial_{R}^{2}}+\frac{N}{2 R} \frac{\partial}{\partial_{R}}+\frac{1}{4 R^{2}} \Delta_{\mathbb{S}},
$$

where $N=p+q$ and $\Delta_{\mathbb{S}}$ is the Laplace operator on $\mathbb{S}^{N-1}$. (It does not look as usual because of the change of $r=|x|$ into $r^{2}=R$.) Since

$$
\Delta_{\mathbb{S}} F_{k}=4 U\left(L_{p, q} P_{k}\right)=-4 k\left(k+\frac{N}{2}-1\right) F_{k},
$$

one may check directly that $H(R, X)=R^{k} F_{k}(X)$ is a solution of $\Delta H=0$.

In other words, the solutions of

$$
\left(\frac{\partial^{2}}{\partial_{R}^{2}}+\frac{N}{2 R} \frac{\partial}{\partial_{R}}+\frac{1}{R^{2}} L_{p, q}\right) F=0
$$

correspond to harmonic functions in $\mathbb{R}^{p} \times \mathbb{R}^{q}$ which are radial in both components (bi-radial harmonic functions). If $(X, Y)$ are the two component of a point in $\mathbb{R}^{p+q}$, then this harmonic function is

$$
\left(|X|^{2}+|Y|^{2}\right)^{k} P_{k}\left(\frac{|X|^{2}-|Y|^{2}}{|X|^{2}+|Y|^{2}}\right) .
$$

Proof. (Of Bateman's formula 5.1.)

Let $L=L_{p, q}$. The function $K(s, t)=P_{k}(s) P_{k}(t)$ is a solution of the wave equation

$$
\left(L_{s}-L_{t}\right) K=0 .
$$

In order to prove the assertion, which amounts to verify the identity of two polynomials, it is enough to check it on an open set. We shall choose to prove it on the set $\{s \in(-1,1), t>1\}$, on which the wave equation $\left(L_{s}-L_{t}\right) K=0$ becomes an elliptic equation.

On the other hand, consider a solution $G(R, x)$ of 


$$
\left(\frac{\partial^{2}}{\partial_{R}^{2}}+\frac{N}{2 R} \frac{\partial}{\partial_{R}}+\frac{1}{R^{2}} L_{p, q}\right) G=0,
$$

and perform the change of variable

$$
R=s+t ; x=\frac{1+s t}{s+t} .
$$

This equation becomes $\left(L_{s}-L_{t}\right) G=0$. (We shall leave the computation to the reader, since it is just brute calculus.)

This strange (and miraculous) change of variables may be much understood if we first operate a change of variables to reduce the leading terms to $\partial_{x}^{2}+\partial_{y}^{2}$ in both equations, and then observe that the transformation we made is conformal in $\mathbb{R}^{2}$, and thus preserves the leading terms. But we could find no simple geometric transformation, even in the case where $p$ and $q$ are positive integers, to understand this change of a bi-Jacobi equation into a bi-radial harmonic function.

Therefore, the right-hand side of Bateman's formula is a solution of the wave equation $\left(L_{s}-L_{t}\right) F=0$. The coefficients $b_{k, r}$ are computed in such a way that the two polynomials coincide on $t=1$.

To see that they must coincide everywhere, it is enough to remark that if two polynomials $A(s, t)$ and $B(s, t)$ in $(s, t)$ are solutions of the wave equation which coincide on $t=1$, they coincide everywhere. Indeed, we may write

$$
A(s, t)=\sum_{r=0}^{k} a_{r} P_{r}(s) P_{r}(t), B(s, t)=\sum_{r=0}^{k} b_{r} P_{k}(s) P_{k}(t),
$$

and identifying the values in $t=1$ produces $a_{r}=b_{r}, r=0, \ldots, k$.

We now turn to the proof of Koornwinder's formula.

We begin with a lemma. Here, we shall use for the first time that $q>p$.

Lemma 5.3. Consider a bi-radial analytic function $H$ on $\mathbb{R}^{p+q}$, that is to say $H(X, Y)=h\left(|X|^{2},|Y|^{2}\right)$ where $h$ is an analytic function on $\mathbb{R}^{2}$. If moreover $H$ is harmonic on $\mathbb{R}^{p+q}$, then it holds

$$
H(X, Y)=\int_{S 0(q)} H(X+\mathrm{i} \pi(R Y), 0) \nu(d R)
$$

where $\nu(d R)$ is the Haar measure on the group $S O(q)$, and $\pi$ is the orthogonal projection from $\mathbb{R}^{q}$ onto $\mathbb{R}^{p}$.

As a consequence, if $H(X, Y)=h\left(|X|^{2},|Y|^{2}\right)$ and $g(x)=h(x, 0)$, then

$$
\begin{aligned}
& h\left(|X|^{2},|Y|^{2}\right)= \\
& \int_{[-1,1]^{2}} g\left(|X|^{2}-\frac{1+u}{2}|Y|^{2}+\mathrm{i} \sqrt{2}|X||Y| \sqrt{1+u} s\right) d \mu_{p, q-p}(u) d \mu_{p-1, p-1}(s) .
\end{aligned}
$$


In practice, we shall just apply this lemma with polynomials functions $h$. Proof. (Of Lemma 5.3.)

The proof comes from the following remark. We observe that if $F$ is any analytic radial function in $\mathbb{R}^{p}$, namely $F(X)=f\left(|X|^{2}\right)$ where $f$ is real analytic, then $F(X+\mathrm{i} Y)$ is a solution in $\mathbb{R}^{p} \times \mathbb{R}^{p}$ of $\Delta_{X} F+\Delta_{Y} F=0$, that is to say that this function is harmonic in $\mathbb{R}^{2 p}$. This is clear if we consider that $F(X+Y)$ is a solution of $\Delta_{X} F=\Delta_{Y} F$.

Remark here that in this analytic continuation, we consider some functions $f\left(|X+\mathrm{i} Y|^{2}\right)$, where

$$
|X+\mathrm{i} Y|^{2}=|X|^{2}-|Y|^{2}+2 \mathrm{i} X \cdot Y .
$$

This is not the norm of $X+\mathrm{i} Y$ considered as a point in $\mathbb{C}^{p}$.

Then, $F(X+\mathrm{i} \pi(Y))$ is harmonic in $\mathbb{R}^{p+q}$, since the projection of the Laplace operator on $\mathbb{R}^{q}$ is the Laplace operator on $\mathbb{R}^{p}$. Hence, for any element $R \in S O(q), F(X+\mathrm{i} \pi(R Y))$ is harmonic in $\mathbb{R}^{p+q}$ since the Laplace operator on $\mathbb{R}^{q}$ is invariant under rotations.

From this, we see that

$$
\tilde{H}(X, Y)=\int_{S O(q)} H(X+\mathrm{i} \pi(R Y), 0) \nu(d R)
$$

is harmonic. Observe also that it is bi-radial. It is obviously radial in $Y$, since we averaged using the Haar measure on $S O(q)$. To see that it is radial in $X$, we just observe that, if $R_{1} \in S O(p)$, one has

$$
H\left(R_{1} X+\mathrm{i} \pi(Z), 0\right)=H\left(X+\mathrm{i} R_{1}^{-1} \pi(Z), 0\right)
$$

since $H(X, 0)$ is radial. Moreover, for any $R_{1} \in S O(p)$, there exists $R_{2} \in$ $S O(q)$ such that $R_{1} \pi(Z)=\pi\left(R_{2} Z\right)$.

Now, let us remark that we will get (12) from (11) just by expliciting the latter formula. For that, we write $X=|X| e_{1}$, where $e_{1} \in \mathbb{S}^{p-1}$ and $\pi(R Y)=|Y| \sqrt{\frac{1+u}{2}} Y_{1}$, where $Y_{1} \in \mathbb{S}^{p-1}$.

We know that if $R$ is chosen according to the Haar measure on $S O(q)$, the law of $R \frac{Y}{|Y|}$ is uniform on $\mathbb{S}^{q}$, and therefore, writing $\pi\left(R \frac{Y}{|Y|}\right)=|Y| \sqrt{\frac{1+u}{2}} Y_{1}$, the law of $\left(x, Y_{1}\right)$ is $\mu_{q-p, p}(d x) \otimes \sigma_{p-1}\left(d Y_{1}\right)$, as we saw at the end of paragraph 5.1. Therefore, if we set $s=e_{1} \cdot Y_{1}$, the law of $(u, s)$ is $\mu_{p, q-p}(d u) \otimes \mu_{p-1, p-1}(d s)$.

Then,

$$
|X+\mathrm{i} \pi(R Y)|^{2}=|X|^{2}-\frac{1+u}{2}|Y|^{2}+\mathrm{i} \sqrt{2}|X||Y| \sqrt{1+u} e_{1} \cdot Y_{1},
$$

and

$$
\begin{aligned}
& \int_{S O(q)} g\left(\left(|X+\mathrm{i} \pi(R Y)|^{2}\right) \nu(d R)=\right. \\
& \int_{[-1,1]^{2}} g\left(|X|^{2}-\frac{1+u}{2}|Y|^{2}+\mathrm{i} \sqrt{2}|X||Y| \sqrt{1+u} s\right) \mu_{p, q-p}(d x) \mu_{p-1, p-1}(d s) .
\end{aligned}
$$


To finish the proof of the first formula (11), we observe that the two members of (11) coincide on $Y=0$. On the other hand, the explicit formulation given in (12) shows that if $g$ is analytic, then the right-hand side in (11) is also analytic in $\left(|X|^{2},|Y|^{2}\right)$. Indeed, if we observe that the measure $\mu_{p-1, p-1}(d s)$ is symmetric, all odd powers of $|Y||X|$ in the polynomial extension of

$$
\left(|X|^{2}-\frac{1+u}{2}|Y|^{2}+\mathrm{i} \sqrt{2}|X||Y| \sqrt{1+u} s\right)^{n}
$$

will disappear through integration. We are therefore left with a series in $\left(|X|^{2},|Y|^{2}\right)$.

It remains to see that two analytic harmonic bi-radial functions which coincide on $Y=0$ coincide everywhere. Let $h\left(|X|^{2},|Y|^{2}\right)$ an analytic bi-radial harmonic function on $\mathbb{R}^{p+q}$. The function $h$ is a solution of

$$
\left(x \partial_{x}^{2}+\frac{p}{2} \partial_{x}+y \partial_{y}^{2}+\frac{q}{2} \partial_{y}\right) h=0 .
$$

Then, we see that if we write the expansion

$$
h(x, y)=\sum_{n, m} a_{n, m} x^{n} y^{m},
$$

one has

$$
a_{n, m+1}=-a_{n+1, m} \frac{(n+1)(n+p / 2)}{(m+1)(m+q / 2)} .
$$

This shows that as soon as one knows $\left(a_{n, 0}\right)$, one knows $h$. This completes the proof of Lemma 5.3.

Proof. (Of Koornwinder's formula (5.2), for $p$ and $q$ integers. )

In the case where $p$ and $q$ are non-negative integer, it turns out that it once again relies on properties of the harmonic functions in the Euclidean space.

First, we lift both members on $\mathbb{R}^{p+q}$ and then we multiply them by $R^{k}$, where $R=|X|^{2}+|Y|^{2}$.

As we have seen before, the function

$$
\left(|X|^{2}+|Y|^{2}\right)^{k} P_{k}\left(\frac{|X|^{2}-|Y|^{2}}{|X|^{2}+|Y|^{2}}\right)
$$

is a bi-radial harmonic function, which is a polynomial in $|X|^{2}$ and $|Y|^{2}$.

It remains to apply Lemma 5.3 to conclude the proof.

If we want to extend the proof of Koornwinder's formula when $p$ and $q$ are no longer integers, then we just have to observe that, setting $S=|X|^{2}$ and $T=|Y|^{2}$, we used the fact that the fact that

$$
H(S, T)=(S+T)^{k} P_{k}\left(\frac{S-T}{S+T}\right)
$$


is a solution on $(0, \infty)^{2}$ of

$$
\left(S \partial_{S}^{2}+\frac{p}{2} \partial_{S}+T \partial_{T}^{2}+\frac{q}{2} \partial_{T}\right) H=0,
$$

and that for any analytic function $f$, the function

$$
K(S, T)=\int f\left(S-\frac{1+u}{2} T+\mathrm{i} \sqrt{2} \sqrt{S T} \sqrt{1+u} s\right) \mu_{p, q-p}(d x) \mu_{p-1, p-1}(d s)
$$

is also a solution of the same equation (but this time, one has to compute that by brute force!).

Remarks. 1. The proof of Koornwinder's formula gives a representation, for analytic functions, of solutions $H(S, T)$ of $L H=0$, where

$$
L=S \partial_{S}^{2}+\frac{p}{2} \partial_{S}+T \partial_{T}^{2}+\frac{q}{2} \partial_{T},
$$

in terms of the boundary values $H(S, 0)$. This is some kind of Poisson formula. In such a formula, one has (at least for bounded functions)

$$
H(x, y)=\mathbb{E}_{x, y}\left(H\left(X_{T}, Y_{T}\right)\right),
$$

where $\left(X_{s}, Y_{s}\right)$ is the diffusion with generator $L$, and $T$ the hitting time of the boundary.

Here, at least when $q \geq 2$, the boundary is polar and the set $\{y=0\}$ is never attained. But the representation is given here through a complex variable (and of course our functions are unbounded). So it happens "as if" the process is willing to hit the boundary, provided one allows complex values (we do not know which is the meaning of that, of course). But it is certainly worth looking for more general integral representations of this type, with complex values on polar sets.

2. Of course, when $q$ converges to $p$, the measure $\mu_{p, q-p}(d s)$ converges to the Dirac mass at the point 1, and Koornwinder's formula of Lemma 5.2 gives for the ultraspherical polynomials

$$
\frac{P_{k}^{p, p}(x)}{P_{k}^{p, p}(1)}=\int_{[-1,1]^{2}}\left[x+\mathrm{i} \frac{\sqrt{1-x^{2}}}{2} v\right]^{k} \mu_{p-1, p-1}(d v) .
$$

One may check directly this formula when $p \in \mathbb{N}$, through a much simpler argument, using harmonic functions in $\mathbb{R}^{p+1}$ instead of harmonic functions in $\mathbb{R}^{2 p}$. This time, one has to extend the polynomial $P_{k}^{p, p}$ into

$$
F_{k}(X)=|X|^{k} P_{k}^{p, p}\left(\frac{X}{|X|} \cdot e_{1}\right)
$$

where $e_{1}$ is any point of the unit sphere. 


\section{References}

1. A. Achour and K. Trimèche. Opérateurs de translation généralisée associés à un opérateur différentiel singulier sur un intervalle borné. C. R. Acad. Sci. Paris Sér. $A$-B, 288(7):A399-A402, 1979.

2. C. Ané, S. Blachère, D. Chafaï, P. Fougères, I. Gentil, F. Malrieu, C. Roberto, and G. Scheffer. Sur les inégalités de Sobolev logarithmiques, volume 10 of Panoramas et Synthèses. Société Mathématique de France, Paris, 2000. With a preface by D. Bakry and M. Ledoux.

3. R. Askey. Jacobi polynomials. I. New proofs of Koornwinder's Laplace type integral representation and Bateman's bilinear sum. SIAM J. Math. Anal., 5:119-124, 1974.

4. R. Askey and J. Fitch. Integral representations for Jacobi polynomials and some applications. J. Math. Anal. Appl., 26:411-437, 1969.

5. D. Bakry. Transformations de Riesz pour les semi-groupes symétriques. II. Étude sous la condition $\Gamma_{2} \geq 0$. In Séminaire de probabilités, XIX, 1983/84, volume 1123 of Lecture Notes in Math., pages 145-174. Springer, Berlin, 1985.

6. D. Bakry. Étude des transformations de Riesz dans les variétés riemanniennes à courbure de Ricci minorée. In Séminaire de Probabilités, XXI, volume 1247 of Lecture Notes in Math., pages 137-172. Springer, Berlin, 1987.

7. D. Bakry. The Riesz transforms associated with second order differential operators. In Seminar on Stochastic Processes, 1988 (Gainesville, FL, 1988), volume 17 of Progr. Probab., pages 1-43. Birkhäuser Boston, Boston, MA, 1989.

8. D. Bakry and M. Echerbault. Sur les inégalités GKS. In Séminaire de Probabilités, XXX, volume 1626 of Lecture Notes in Math., pages 178-206. Springer, Berlin, 1996.

9. D. Bakry and M. Emery. Diffusions hypercontractives. In Séminaire de probabilités, XIX, 1983/84, pages 177-206. Springer, Berlin, 1985.

10. W. Beckner. Inequalities in Fourier analysis. Ann. of Math. (2), 102(1):159-182, 1975.

11. W. Beckner. Sobolev inequalities, the Poisson semigroup, and analysis on the sphere $S^{n}$. Proc. Nat. Acad. Sci. U.S.A., 89(11):4816-4819, 1992.

12. N. H. Bingham. Random walk on spheres. Z. Wahrscheinlichkeitstheorie und Verw. Gebiete, 22:169-192, 1972.

13. H. Bloom, W.R. et Heyer. Harmonic analysis of probability measures on hypergroups. Walter de Gruyter, 1995.

14. B. L. J. Braaksma and B. Meulenbeld. Jacobi polynomials as spherical harmonics. Nederl. Akad. Wetensch. Proc. Ser. A $71=$ Indag. Math., 30:384-389, 1968.

15. H. Brézis. Analyse fonctionnelle. Masson, Paris, 1983. Théorie et applications.

16. H. Chebli. Opérateurs de translation généralisée et semi-groupes de convolution. In Théorie du potentiel et analyse harmonique (Journées Soc. Math. France, Inst. Recherche Math. Avancée, Strasbourg, 1973), pages 35-59. Lecture Notes in Math., Vol. 404. Springer, Berlin, 1974.

17. W. C. Connett, C. Markett, and A. L. Schwartz. Jacobi polynomials and related hypergroup structures. In Probability measures on groups, X (Oberwolfach, 1990), pages 45-81. Plenum, New York, 1991.

18. W. C. Connett and A. L. Schwartz. Product formulas, hypergroups, and the Jacobi polynomials. Bull. Amer. Math. Soc. (N.S.), 22(1):91-96, 1990. 
19. W. C. Connett and A. L. Schwartz. Subsets of $\mathbf{R}$ which support hypergroups with polynomial characters. In Proceedings of the International Conference on Orthogonality, Moment Problems and Continued Fractions (Delft, 1994), volume 65, pages 73-84, 1995.

20. E. B. Davies. Heat kernels and spectral theory. Cambridge University Press, Cambridge, 1990.

21. C. Dellacherie and P. A. Meyer. Probabilités et potentiel. Hermann, Paris, 1975. Chapitres I à IV, Édition entièrement refondue, Publications de l'Institut de Mathématique de l'Université de Strasbourg, No. XV, Actualités Scientifiques et Industrielles, No. 1372.

22. P. Diaconis. Group representations in probability and statistics. Institute of Mathematical Statistics, Hayward, CA, 1988.

23. A. Dijksma and T. H. Koornwinder. Spherical harmonics and the product of two Jacobi polynomials. Nederl. Akad. Wetensch. Proc. Ser. A $\mathbf{7 4 = I n d a g . ~ M a t h . , ~}$ 33:191-196, 1971.

24. G. Gasper. Linearization of the product of Jacobi polynomials. Can. J. Math. 22:171-175,582-593, 1970 .

25. G. Gasper. Positivity and the convolution structure for Jacobi series. Ann. of Math., 2(93):112-118, 1971.

26. G. Gasper. Banach algebras for Jacobi series and positivity of a kernel. Ann. of Math., 2(95):261-280, 1972.

27. R.B. Griffiths. Correlation in Ising ferromagnets. J. Math.Phys., 8:478-489, 1967.

28. L. Gross. Logarithmic Sobolev inequalities. Amer. J. Math., 97(4):1061-1083, 1975.

29. L. Gross. Logarithmic Sobolev inequalities and contractivity properties of semigroups. In Dirichlet forms (Varenna, 1992), pages 54-88. Springer, Berlin, 1993.

30. J. Hadamard. Résolution d'une question relative aux déterminants. Bulletin des Sciences Mathématiques, 17:240-246, 1893.

31. I. Martin Isaacs. Character theory of finite groups. Dover Publications Inc., New York, 1994. Corrected reprint of the 1976 original [Academic Press, New York; MR0460423 (57 \#417)].

32. D.G. Kelly and S. Sherman. General Griffiths' inequality on correlation in Ising ferromagnets. J. Math.Phys., 9:466-484, 1968.

33. H. Kharaghani and B. Tayfeh-Rezaie. A Hadamard matrix of order $428 . \mathrm{J}$. Combin. Des., 13(6):435-440, 2005.

34. T. H. Koornwinder. Jacobi polynomials. II. An analytic proof of the product formula. SIAM J. Math. Anal., 5:125-137, 1974.

35. T.H. Koornwinder. The addition formula for Jacobi polynomials and spherical harmonics. SIAM J. Appl. Math., 25:236-246, 1973. Lie algebras: applications and computational methods (Conf., Drexel Univ., Philadelphia, Pa., 1972).

36. O. Mazet. Semigroupes de Markov associés à une famille de polynômes orthogonaux. PhD thesis, Université Paul Sabatier, Jan 1998.

37. R. E. A. C. Paley. On orthogonal matrices. Journal of Mathematics and Physics, 12:311-320, 1933.

38. G. Scheffer. Local Poincaré inequalities in non-negative curvature and finite dimension. J. Funct. Anal., 198(1):197-228, 2003.

39. G. Szegö. Orthogonal Polynomials. American Mathematical Society, 4th edition, 1975. 
40. A. Zettl. Sturm-Liouville theory, volume 121 of Mathematical Surveys and Monographs. American Mathematical Society, Providence, RI, 2005. 